\title{
Mapping subnational HIV mortality in six Latin American countries with incomplete vital registration systems
}

\author{
Local Burden of Disease HIV Collaborators
}

\begin{abstract}
Background: Human immunodeficiency virus (HIV) remains a public health priority in Latin America. While the burden of HIV is historically concentrated in urban areas and high-risk groups, subnational estimates that cover multiple countries and years are missing. This paucity is partially due to incomplete vital registration (VR) systems and statistical challenges related to estimating mortality rates in areas with low numbers of HIV deaths. In this analysis, we address this gap and provide novel estimates of the HIV mortality rate and the number of HIV deaths by age group, sex, and municipality in Brazil, Colombia, Costa Rica, Ecuador, Guatemala, and Mexico.

Methods: We performed an ecological study using VR data ranging from 2000 to 2017, dependent on individual country data availability. We modeled HIV mortality using a Bayesian spatially explicit mixed-effects regression model that incorporates prior information on VR completeness. We calibrated our results to the Global Burden of Disease Study 2017.

Results: All countries displayed over a 40-fold difference in HIV mortality between municipalities with the highest and lowest age-standardized HIV mortality rate in the last year of study for men, and over a 20-fold difference for women. Despite decreases in national HIV mortality in all countries - apart from Ecuador-across the period of study, we found broad variation in relative changes in HIV mortality at the municipality level and increasing relative inequality over time in all countries. In all six countries included in this analysis, 50\% or more HIV deaths were concentrated in fewer than $10 \%$ of municipalities in the latest year of study. In addition, national age patterns reflected shifts in mortality to older age groups—the median age group among decedents ranged from 30 to 45 years of age at the municipality level in Brazil, Colombia, and Mexico in 2017.

Conclusions: Our subnational estimates of HIV mortality revealed significant spatial variation and diverging local trends in HIV mortality over time and by age. This analysis provides a framework for incorporating data and uncertainty from incomplete VR systems and can help guide more geographically precise public health intervention to support HIV-related care and reduce HIV-related deaths.
\end{abstract}

Keywords: HIV/AIDS, Latin America, HIV mortality, Vital registration, Small area estimation, Mapping, Spatial statistics

Correspondence: ladwyer@uw.edu

Institute for Health Metrics and Evaluation, University of Washington, Seattle, WA, USA

(c) The Author(s). 2021 Open Access This article is licensed under a Creative Commons Attribution 4.0 International License, which permits use, sharing, adaptation, distribution and reproduction in any medium or format, as long as you give appropriate credit to the original author(s) and the source, provide a link to the Creative Commons licence, and indicate if changes were made. The images or other third party material in this article are included in the article's Creative Commons licence, unless indicated otherwise in a credit line to the material. If material is not included in the article's Creative Commons licence and your intended use is not permitted by statutory regulation or exceeds the permitted use, you will need to obtain permission directly from the copyright holder. To view a copy of this licence, visit http://creativecommons.org/licenses/by/4.0/ The Creative Commons Public Domain Dedication waiver (http://creativecommons.org/publicdomain/zero/1.0/) applies to the data made available in this article, unless otherwise stated in a credit line to the data. 


\section{Background}

Human immunodeficiency virus (HIV) continues to be a large contributor to morbidity and mortality across the globe $[1,2]$. While the burden of the HIV epidemic is most concentrated in sub-Saharan Africa, HIV remains a public health priority in Latin America. In 2017, the Global Burden of Disease Study (GBD) estimated over 30,000 deaths from HIV/AIDS-related causes in the region $[1,2]$. To combat the epidemic, the Joint United Nations Program on HIV/AIDS (UNAIDS) Fast-Track strategy emphasizes the need to reduce HIV-related deaths to less than 500,000 deaths worldwide by 2020-a $75 \%$ reduction from deaths in 2010 [3]. UNAIDS further targets a 90\% reduction in HIV-related deaths by 2030 [3]. Despite increased access to antiretroviral therapy in many countries in Latin America [3, 4], few countries show substantial reduction in HIV mortality since 2000 $[1,2]$. Continued efforts are needed to track progress towards meeting the UNAIDS Fast-Track goals with respect to HIV mortality.

Country-level estimates of HIV mortality in Latin America are available from a variety of sources $[1,5]$ and estimates of mortality exist at the state level in select countries such as Brazil and Mexico [1]. Beyond this, however, detailed subnational estimates at the second administrative level in many countries in Latin America are absent. This lack of subnational estimates is alarming given the localized burden of HIV in urban areas and among high-risk subgroups such as people who inject drugs, sex workers, and men who have sex with men (MSM) [6-10]. Additionally, inequalities in HIV burden across local geographies likely occur given that many underlying drivers of HIV infection and death-such as poverty, incarceration, undernutrition, distribution of health practitioners, and access to health services-vary across geographic areas and through time [11-14]. Previous studies have confirmed substantial within-country variation in mortality rates but are limited to select countries, areas, and years [15-18].

The paucity of evidence on subnational HIV mortality is likely due to several methodological challenges associated with a granular spatial modeling of HIV mortality in Latin American countries. Deaths attributable to HIV are inherently small in number in areas with small populations, adding stochastic noise to direct estimates [19]. Past approaches have used Bayesian models that apply small area methods that borrow strength across age, time, and space to produce stable estimates of mortality rates in areas with a small number of deaths [15, 20-22]. An additional complication in HIV mortality estimation is that-due to stigma or misdiagnosis-HIV deaths may be misclassified and coded to other underlying causes of death such as tuberculosis, endocrine disorders, meningitis, or encephalitis [23-25]. Moreover, vital registration
(VR) systems in many countries in Latin America are incomplete and not all deaths are recorded in official statistics [23, 25-27]. While a variety of methods have been proposed to estimate the completeness of death registration at the country level [28, 29], standard methods rely on stable population and sex pattern assumptions that often do not hold in small subnational areas [29, 30]. To resolve the difficulties associated with small numbers of deaths and VR completeness, Schmertmann and Gonzaga proposed a Bayesian model framework for small area life expectancy estimation in countries with incomplete VR systems [31]. This method incorporates a novel functional form for mortality that is informed by prior distributions for VR completeness coverage based on empirical evidence.

In this analysis, we address these challenges by utilizing comprehensive cause of death assignment and applying a small area estimation framework that incorporates prior information on VR completeness to produce estimates of HIV mortality and deaths due to HIV by age and sex at the municipality level in six countries in Latin America: Brazil, Mexico, Guatemala, Costa Rica, Colombia, and Ecuador. Our modeling approach leverages information from neighboring areas across space and time to produce estimates across all years of available data. These six countries were selected based on availability of VR data, but not all contained the same range of available years: our analysis extends from 2000 to 2017 in Brazil, Colombia, and Mexico; from 2009 to 2017 in Guatemala; from 2004 to 2014 in Ecuador; and from 2014 to 2016 in Costa Rica.

\section{Methods \\ Overview}

This analysis complied with the Guidelines for Accurate and Transparent Health Estimates Reporting (GATHER) [32]. Our ecological study estimated the HIV mortality rate and the number of HIV deaths by age group and sex at the municipality level for all years of available VR (Additional file 1: Figure S1). All analyses were carried out at the second administrative unit level, which we refer to as municipalities for convenience unless referencing country-specific results, where we use the appropriate national nomenclature for the administrative subdivision (municipality for Brazil, Colombia, Guatemala, Mexico; canton for Costa Rica and Ecuador). Municipalities were combined as needed to create stable units of analysis over the study period, reducing the total number of areas analyzed in select countries (Table 1; Additional file 1: Table S1). In the results, all presented rates are age-standardized for comparison between countries, unless otherwise stated. We used standard age weights produced by GBD 2017 for age standardization [1]. 
Table 1 Data availability and administrative characteristics by country

\begin{tabular}{lllll}
\hline Country & Years of available VR data & $\begin{array}{l}\text { Name of second } \\
\text { administrative } \\
\text { subdivision }\end{array}$ & $\begin{array}{l}\text { Total number of second } \\
\text { administrative subdivisions }\end{array}$ & $\begin{array}{l}\text { Number of modeled units } \\
\text { in this analysis }\end{array}$ \\
\hline Brazil & $2000-2017$ & Municipality & 5570 & 5477 \\
Colombia & $2000-2017$ & Municipality & 1122 & 1115 \\
Costa Rica & $2014-2016$ & Canton & 81 & 81 \\
Ecuador & $2004-2014$ & Canton & 224 & 222 \\
Guatemala & $2009-2017$ & Municipality & 340 & 333 \\
Mexico & $2000-2017$ & Municipality & 2458 & 2441 \\
\hline
\end{tabular}

\section{Data}

\section{Vital registration data}

Vital registration (VR) mortality data consisted of anonymized individual-level records from all deaths reported in each country's VR system occurring between the years of study (Additional file 1: Table S2). These records were tabulated by municipality of residence, age group (0-4, $5-9,10-14, \ldots, 75-79$, and $\geq 80$ years), sex, and the underlying cause of death according to the tenth revision of the International Classification of Diseases (ICD-10) [33]. We standardized VR data using methods developed for the GBD [1]. This process requires all deaths to be attributed to a single underlying cause of death following ICD guidelines and fits within a hierarchy of mutually exclusive and collectively exhaustive causes. Deaths that were coded with ICD-10 codes that could not be an underlying cause of death, as well as deaths that were coded to non-specific causes of death, were redistributed to most detailed causes of death by age, sex, municipality, and year according to a framework developed by Naghavi et al. [34] and updated for GBD 2017 [1]. This includes an HIV correction step that rectifies deaths assigned to comorbidities such as tuberculosis, endocrine disorders, meningitis, or encephalitis that diverge from locations without HIV epidemics [1].

\section{Location hierarchy}

We created country-specific location hierarchies that list all subnational administrative units for each year in the specified time period and match each corresponding death from the VR system to the municipality level (Additional file 1: Figure S2). For each country, municipalities were geo-matched to shapefiles provided by the Global Administrative Unit Layers (GAUL) [35] (Brazil, Costa Rica, Guatemala) or the Humanitarian Data Exchange [36] (Colombia, Ecuador, Mexico). In all selected countries, municipality boundaries changed over time, reflecting new boundary designations across the years of study (Table 1). Municipalities that underwent a boundary change during the period of the analysis were merged to create a stable unit across the period of observation, and the municipality-level shapefiles were manually edited to match the split hierarchy using ArcMap version 10.6 [37]. Merged units that included multiple municipalities were modeled as one area, and in the results share the same estimates of HIV mortality rate. Details of these shifts are provided in Additional file 1: Table S1.

\section{Covariates and population}

We included several available covariates to help inform estimates of HIV mortality: population density [38], night-time light brightness [39], urbanicity [40], and travel time to the nearest settlement of more than 50,000 inhabitants [41] (Additional file 1: Table S3). These covariates were selected because they are factors or proxies for factors previously identified in the literature as associated (not necessarily causally) with HIV mortality. Specifically, these four variables were included as measures or proxies for connectedness and urbanicity as HIV historically spread among high-risk groups in urban areas $[6,42]$ and is typically found to be higher in more urban compared to more rural locations. Each covariate was obtained in a raster format at a $5 \times 5-\mathrm{km}$ resolution and required aggregation to the modeled municipality level for inclusion in our modeling framework. This aggregation was done fractionally: raster cells that crossed municipality borders were fractionally allocated to municipalities in proportion to the covered area.

We created age- and sex-specific populations for each municipality unit by aggregating the WorldPop [38] raster to the modified shapefile, utilizing the same fractional aggregation process. The age- and sex-specific populations for municipalities were then scaled to the national population estimates derived from the GBD [1]. To do so, for each country, sex, age group, and year, we defined a population raking factor as the ratio of the GBD population estimate for that same sex, age group, and year to the sum of the WorldPop population for all municipalities within the country, and then multiplied the WorldPop population estimates for each municipality within the country by this raking factor. This resulted in age- and sex-specific population estimates for each 
municipality which aligned with the GBD national population sizes and structures.

\section{Statistical model Vital registration completeness}

Expanding on previous literature [31], we used a Bayesian framework that bypasses a lack of identifiability between the mortality rate and completeness estimate by incorporating an informed prior on VR completeness. In this analysis, we incorporated information from GBD [1] on subnational (for Brazil and Mexico) and national VR completeness (for remaining countries) as well as geographic patterns in under- 5 VR completeness from past analyses [43] to generate priors on municipality-level VR coverage by two age groups $(<15$ years and $15+$ years $)$ and year (Additional file 1: Figure S3). We selected these two age groups based on the available national VR completeness estimated in GBD and established literature and expert opinion [31,44]. The supplemental methods outlined in Additional file 1 summarize our process for generating informed priors on VR completeness in greater detail.

We did not model VR completeness for adults if national GBD completeness estimates exceeded $95 \%$ in all years of available VR data (Costa Rica and Colombia). Similarly, we did not model under-15 VR completeness if GBD estimates of child completeness were greater than $90 \%$ in all years of available VR data (Costa Rica, Guatemala, Mexico). We therefore modeled adult completeness in Ecuador, Guatemala, Mexico, and Brazil, and child completeness in Ecuador, Colombia, and Brazil.

\section{Modeling framework}

We estimated HIV mortality separately by sex using a small area estimation framework built upon a model developed in prior modeling studies $[15,45]$. This Bayesian hierarchical generalized linear model used a Poisson data likelihood to model the number of HIV deaths in a municipality, year, and age group (supplemental methods in Additional file 1). The Poisson distribution was characterized by a parameter that multiplied the mortality rate, and population by municipality and VR completeness by municipality (Colombia, Ecuador, and Guatemala) or state (Brazil and Mexico). Completeness priors added probabilistic information about VR coverage that allowed estimation of mortality rates given counts of registered deaths. We modeled the log of the mortality rate as a linear combination of terms including random effects with conditional autoregressive distributions to smooth over age, year, and municipality. We also included covariates as fixed effects (see supplemental methods in Additional file 1).

Models were estimated separately for each country and sex and fit using the TMB package [46]. One thousand draws were sampled from the approximated posterior distributions of each modeled parameter and used to construct 1000 draws of HIV mortality $\left(m_{j, t, a}\right)$ for each municipality $j$, year $t$, and age group $a$. We calculated point estimates from the mean of these draws, and the lower and upper bounds of the 95\% uncertainty interval from the 2.5th and 97.5th percentiles, respectively, for each age, sex, year, and municipality. Municipality-level estimates for each age, year, and sex were aggregated to the state and national level using a population-weighted average. In Brazil and Mexico, estimates were calibrated to GBD at the state level and estimates were calibrated to national HIV mortality estimates for the remaining four countries. To accomplish this, we calculated the ratio of the national- or state-level estimate from GBD to the mean national estimate derived from population-weighting $m_{j, t, a}$, and multiplied all draws of $m_{j, t}, a$ by this ratio. We generated the number of HIV deaths for each age-sex-year-municipality by multiplying the mean, lower, and upper bounds of our mortality estimates by the corresponding WorldPop population estimate. We quantified the relative inequality as the mortality rate ratios for municipalities in the 90th percentile versus those in the 10th percentile of mortality rate by year. We calculated the absolute inequality as the difference in mortality between municipalities within a country in the 90th percentile and those in the 10th percentile in terms of mortality rate by year. Throughout our analysis, we qualify statements as statistically significant if the posterior probability of that statement exceeds $95 \%$. We completed our analysis using $\mathrm{R}$ version 3.6 .3 [47].

\section{Model assessment}

To assess if including VR completeness in our statistical framework improved model estimates, for the five countries where we applied completeness priors to either children under- 15 or adults we also fit a model where the completeness term in the statistical model, $\pi_{k, t, a^{*}}$, was removed. We then compared the ratio of annual national HIV mortality in children under 15 and adults from GBD to 1000 draws of national estimates of annual HIV mortality from the standard and completeness models. This ratio, known as the raking factor, is plotted in Additional file 1: Figure S4-S9. A raking factor closer to 1 indicates better alignment with GBD, and inclusion of completeness priors generally resulted in closer alignment with national GBD mortality estimates.

\section{Results}

Geographic patterns in HIV mortality rate and notable time trends

Brazil

In Brazil, the estimated national HIV mortality rate for both sexes combined in 2017 was 6.5 (95\% uncertainty interval 6.4-6.7) deaths per 100,000, 8.7 [8.5-8.9] deaths 
among men, and 4.5 [4.4-4.6] deaths among women (Additional file 1: Table S4). Estimated HIV mortality for men in 2017 varied over 53-fold among municipalities: from $0.9(0.2-2.9)$ deaths per 100,000 in the Jordão municipality, Acre state to 47.8 (36.4-61.3) deaths per 100,000 in the Tramandaí municipality, Rio Grande do Sul state (Fig. 1 and Additional file 1: Figure S10). Estimated female HIV mortality in 2017 ranged from 0.8
(0.3-1.7) deaths per 100,000 in the Maraã municipality, Amazonas state to 28.6 (20.7-37.7) deaths per 100,000 in the Tramandaí municipality, Rio Grande do Sul state. Between 2000 and 2017, estimated national HIV mortality decreased by $25.3 \%$ (from 11.7 [11.4-11.9] deaths per 100,000 in 2000) among men and $14.9 \%$ (from 5.3 [5.15.4] deaths per 100,000 in 2000) among women. These national decreases hide substantial variation at the

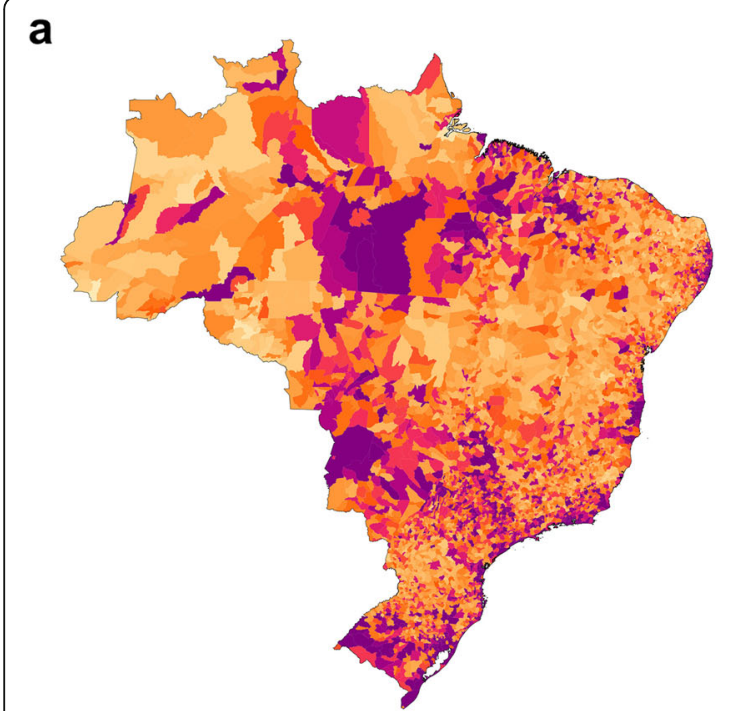

C
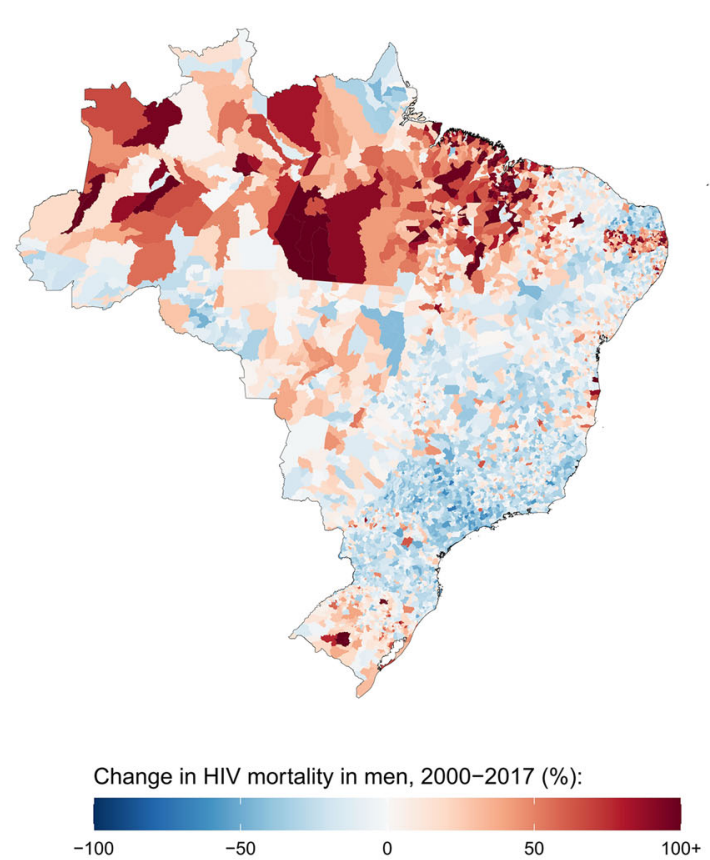

d

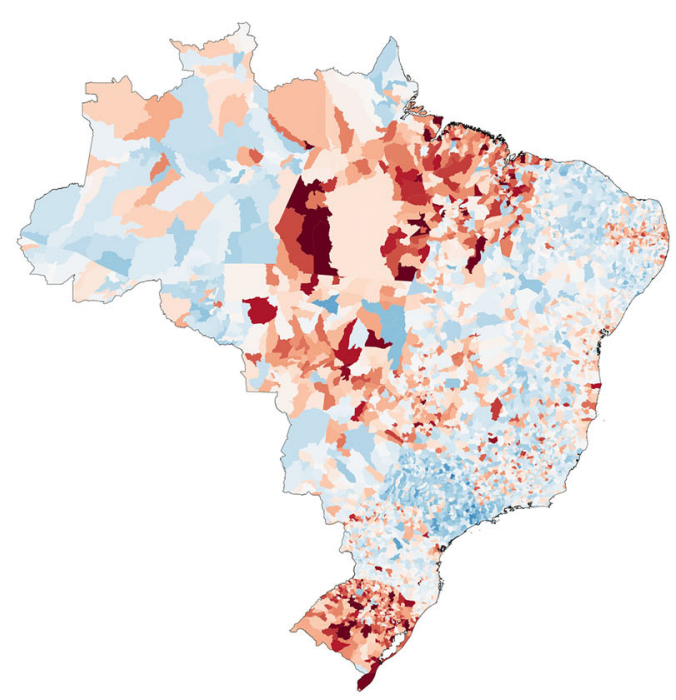

Change in HIV mortality in women, 2000-2017 (\%):

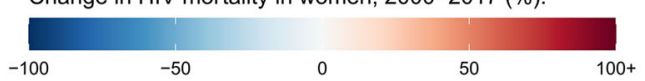

Fig. 1 HIV mortality among men and women in Brazil by municipality, 2017. HIV mortality per 100,000 by municipality in Brazil in 2017 among men (a) and women (b). Relative change in HIV mortality between 2000 and 2017 among men (c) and women (d) 
municipality level. Estimated male HIV mortality decreased in $3389(60.8 \%)$ municipalities, and 91 [1.6\%] municipalities had a statistically significant decrease in HIV mortality. Estimated female HIV mortality decreased in 3000 (53.9\%) municipalities and 37 [0.7\%] municipalities had a statistically significant decrease in HIV mortality. Changes in estimated male HIV mortality at the municipality level ranged from a $248.1 \%$ increase in Bacabal municipality, Maranhão state (from 7.2 [4.910.5] deaths per 100,000 in 2000 to 25.1 [18.9-33.2] deaths per 100,000 in 2017) to a $70.9 \%$ decrease in Ribeirao Preto municipality, São Paulo state (from 32.6 [28.6-36.9] deaths per 100,000 in 2000 to 9.5 [8.1-11.2] deaths per 100,000 in 2017). Changes in estimated HIV mortality among women at the municipality level varied from a $233.9 \%$ increase in Novo Hamburgo municipality, Rio Grande do Sul state (from 5.2 [3.9-6.9] deaths per 100,000 in 2000 to 17.4 [13.8-21.7] deaths per 100,000 in 2017) to a $68.8 \%$ decrease in Jundiaí municipality, São Paulo state (from 5.7 [4.4-7.4] deaths per 100,000 in 2000 to 1.8 [1.2-2.5] deaths per 100,000 in 2017).

\section{Colombia}

In 2017, Colombia's estimated national HIV mortality rate for both sexes combined was $5.0(4.8-5.3)$ deaths per 100,000 (7.8 [7.5-8.1] deaths for men, and 2.5 [2.32.7] deaths for women). Estimated male HIV mortality in 2017 varied over 75 -fold: from $0.5(0.2-0.9)$ deaths per 100,000 in the La Calera municipality, Cundinamarca department to $37.6(26.4-50.9)$ deaths in the Chinchiná municipality, Caldas department (Fig. 2 and Additional file 1: Figure S11). Among women, estimated mortality varied over 86 -fold, fluctuating from 0.2 (0.1$0.5)$ deaths per 100,000 in the La Calera municipality, Cundinamarca department to $17.2(10.6-27.4)$ deaths in the La Virginia municipality, Risaralda department. Between 2000 and 2017, changes in national HIV mortality diverged by sex: estimated HIV mortality decreased among men by $19.8 \%$ (from 9.8 [9.3-10.2] deaths per 100,000 in 2000) but increased among women by $19.5 \%$ (from 2.1 [1.9-2.3] deaths per 100,000 in 2000). While national estimated HIV prevalence decreased among men and not women, HIV mortality increased in a majority of municipalities from 2000 to 2017 for both men and women. Estimated male HIV mortality increased in 594 (60.8\%) municipalities, and five (1.6\%) municipalities had a statistically significant increase in HIV mortality. Estimated female HIV mortality increased in 1081 (96.3\%) municipalities and five (0.4\%) municipalities had a statistically significant increase in HIV mortality. There was large variation in subnational changes in HIV mortality: estimated male HIV mortality ranged from a 361.7\% increase in San Andrés de Tumaco municipality, Nariño department (from 2.8 [1.7-4.3] deaths per 100,
000 in 2000 to 12.8 [9.4-17.1] deaths per 100,000 in 2017) to a $59.7 \%$ decrease in Barbosa municipality, Antioquia department (from 15.5 [9.4-24.5] deaths per 100, 000 in 2000 to 6.2 [3.2-10.5] deaths per 100,000 in 2017). Among women, estimated relative change in HIV mortality at the municipality level varied from a $170 \%$ increase in Puerto Colombia municipality, Atlántico department (from 3 [1.5-5.4] deaths per 100,000 in 2000 to 8.2 [3.9-16.1] deaths per 100,000 in 2017) to a $31.2 \%$ decrease in Bogotá, D.C. municipality, Bogotá, D.C. department (from 1.6 [1.4-1.9] deaths per 100,000 in 2000 to 1.1 [0.9-1.3] deaths per 100,000 in 2017).

\section{Costa Rica}

Among the six countries considered, Costa Rica had the lowest HIV mortality rates in the latest year of study, with an estimated national HIV mortality rate for both sexes combined in 2016 of $3.2(2.5-3.9)$ deaths per 100, 000 (4.9 [4.2-5.8] deaths for men, and 1.5 [1.1-2.3] deaths for women). At the canton level, we estimated male HIV mortality varied over 104-fold in 2016, from $0.5(0.01-2.9)$ deaths per 100,000 in the Aserrí canton to 52.1 (42.1-63.2) deaths in the San José canton, San José province (Fig. 3 and Additional file 1: Figure S12). For women, mortality ranged over 40-fold, from $0.2(0.0-$ 1.5) deaths per 100,000 in the Atenas canton, Alajuela province, to $11.9(7.8-17.4)$ deaths per 100,000 in the San José canton, San José province. Between 2014 and 2016, estimated national HIV mortality decreased by 18.5\% among men (from 6.0 [5.1-7.1] deaths per 100, 000 in 2014) and by $20 \%$ among women (from 1.9 [1.42.7] deaths per 100,000 in 2014). Estimated HIV mortality decreased in $80(98.8 \%)$ municipalities for men and all 81 municipalities for women, though no canton for either sex registered a statistically significant decrease in HIV mortality. National temporal decreases were largely driven by the San José canton, San José province, which had the largest decrease in estimated male HIV mortality: $21.1 \%$, from 65.9 [53.6-81.5] deaths per 100,000 in 2014 to 52.1 [42.1-63.2] deaths per 100,000 in 2016. From 2014 to 2016, estimated HIV mortality in San José canton, San José province, decreased among women by $22.7 \%$ (from 15.3 [10.4-22.9] deaths per 100,000 in 2014 to 11.9 [7.8-17.4] deaths per 100,000 in 2016).

\section{Ecuador}

In 2014, Ecuador had the highest estimated national HIV mortality rate for both sexes combined among the six countries investigated: $7.0(6.5-7.6)$ deaths per 100 , 000 (10.9 [10.1-11.7] deaths for men, and 3.4 [3.0-3.8] deaths for women). Estimated male HIV mortality varied among cantons from $1.1(0.4-2.4)$ deaths per 100,000 in the Tulcán canton, Carchi province, to 50.7 (29.0-81.6) deaths per 100,000 in the Palestina canton (Fig. 4 and 


\section{a}

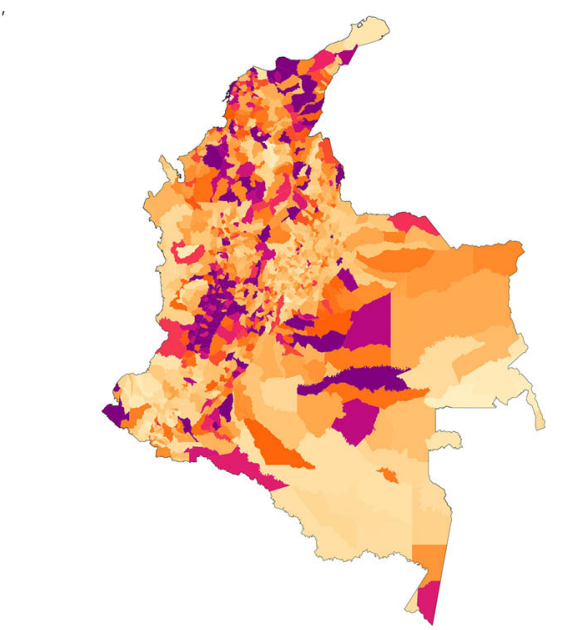

HIV mortality in 2017, men (per 100k)

0

b

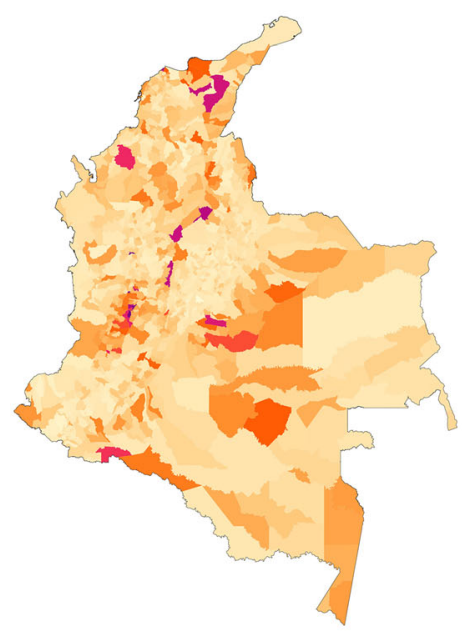

HIV mortality in 2017, women (per 100k)

0
C

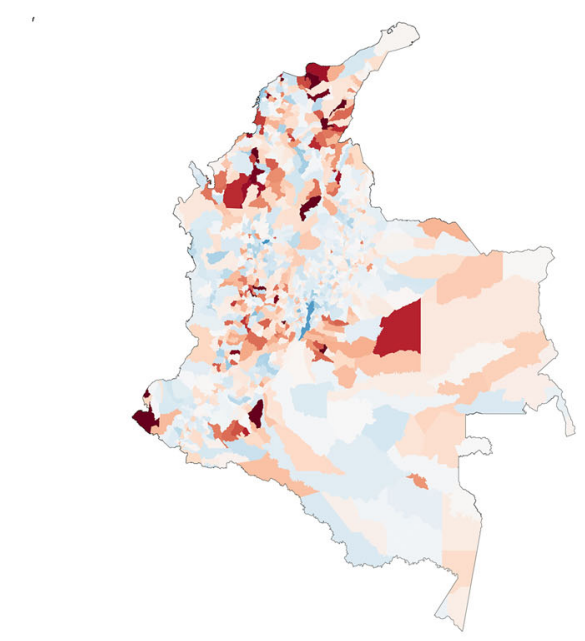

Change in HIV mortality in men, 2000-2017 (\%):

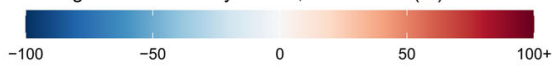

d
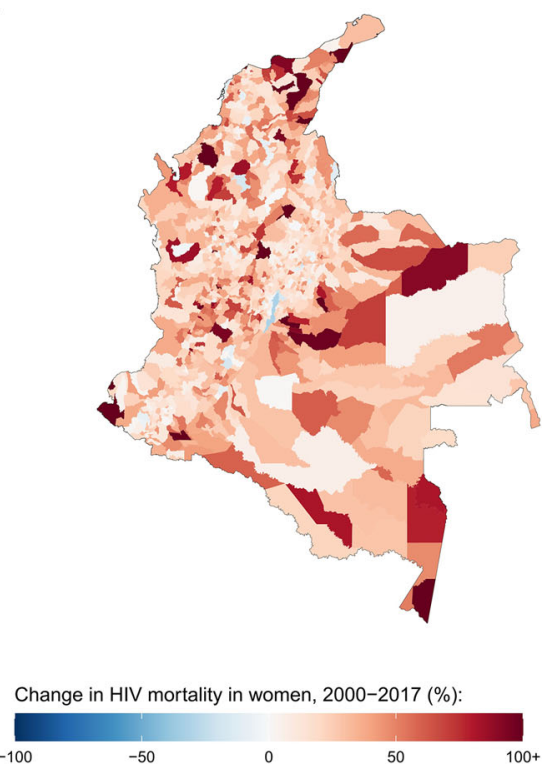

Fig. 2 HIV mortality among men and women in Colombia by municipality, 2017. HIV mortality per 100,000 by municipality in Colombia in 2017 among men (a) and women (b). Relative change in HIV mortality between 2000 and 2017 among men (c) and women (d)

Additional file 1: Figure S16). For women, estimated HIV mortality ranged from $0.6(0.2-1.3)$ deaths per 100 , 000 in the Tulcán canton, Carchi province, to 13.0 (4.329.8) deaths per 100,000 in the San Lorenzo canton, Esmeraldas province. Between 2004 and 2014, estimated national HIV mortality increased by $28.3 \%$ among men (from 8.5 [7.8-9.2] deaths per 100,000 in 2004) and increased by $63.2 \%$ among women (from 2.1 [1.8-2.4] deaths per 100,000 in 2004). Estimated male HIV mortality increased in 216 (96.4\%) cantons, and three [1.3\%] municipalities had a significant increase in HIV mortality. Estimated female HIV mortality increased in 223 (99.6\%) municipalities and one [0.4\%] canton had a statistically significant increase in HIV mortality. Among men, estimated change in HIV mortality at the canton level ranged from a $239.7 \%$ increase in Río Verde canton, Esmeraldas province (from 9.1 [2.9-24.4] deaths per 100,000 in 2004 to 31 [9.5-79.1] deaths per 100,000 in 2014 ), to a $42.7 \%$ decrease in Huaquillas canton, El Oro province (from 8.3 [3.9-15.9] deaths per 100,000 in 2004 


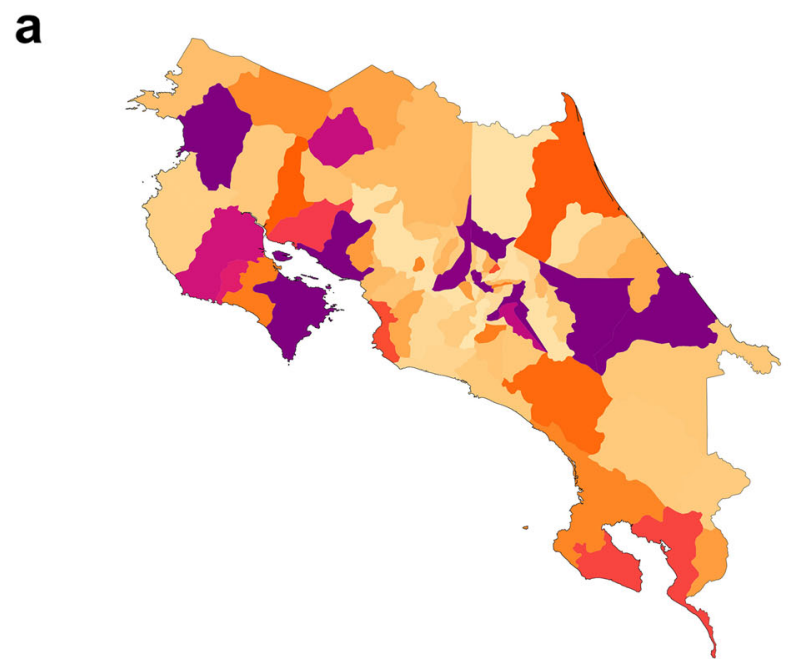

C

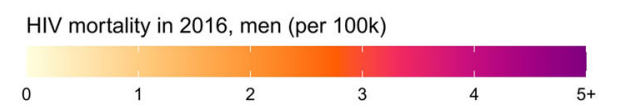

b

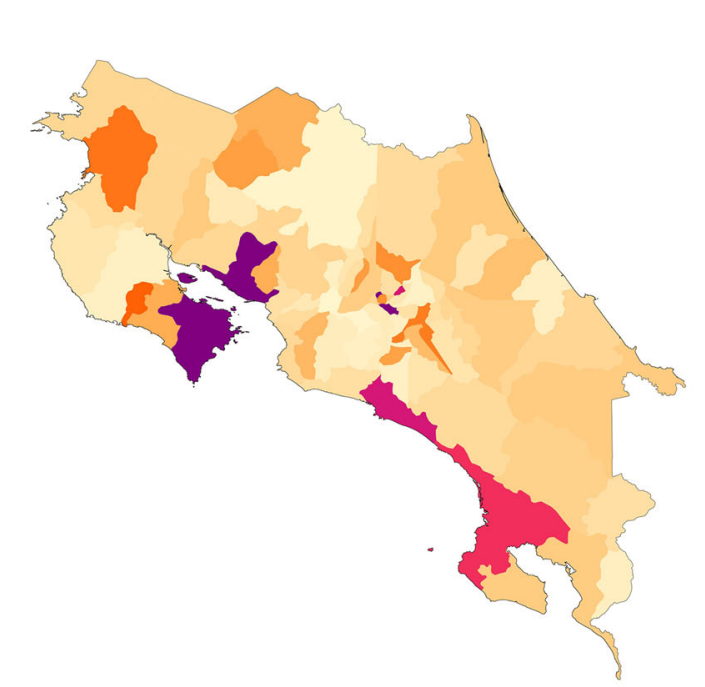

d

HIV mortality in 2016, women (per 100k)

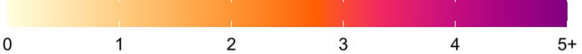

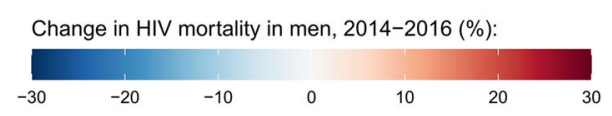
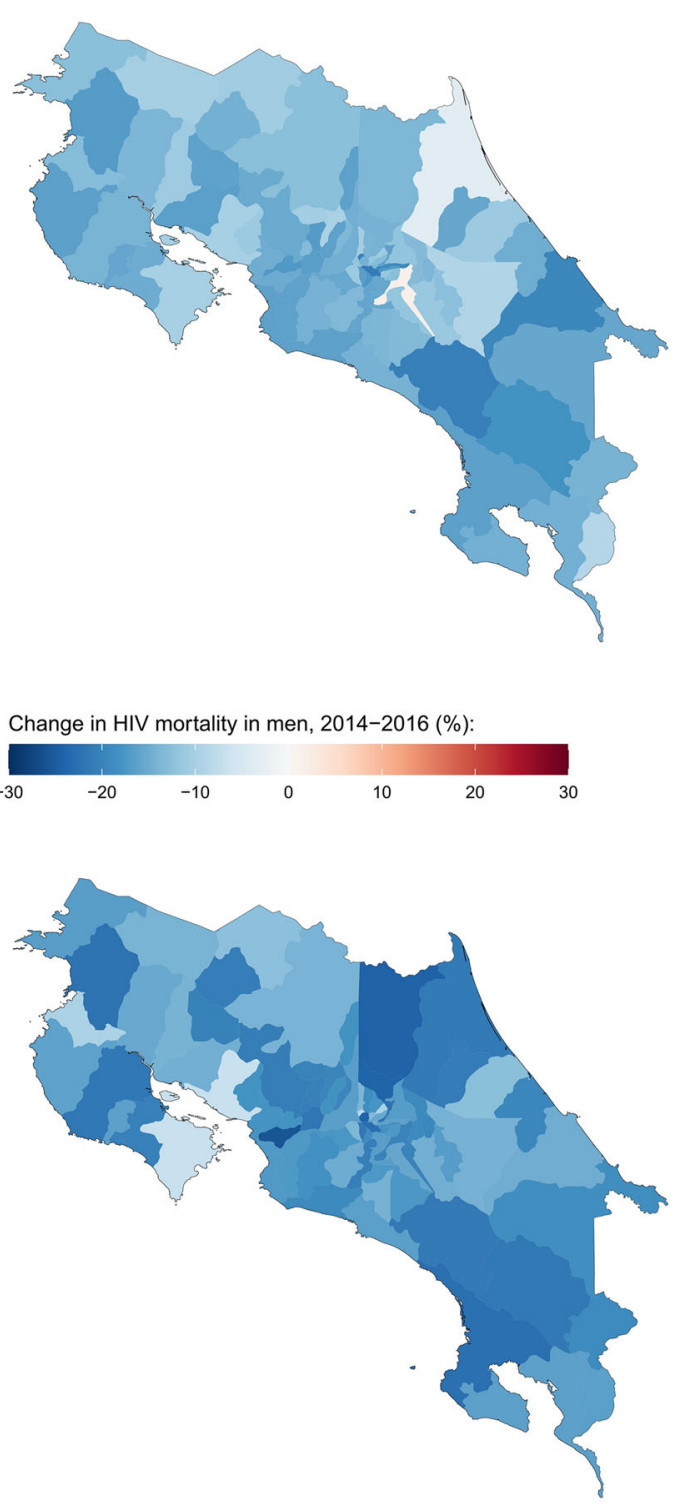

Change in HIV mortality in women, 2014-2016 (\%):

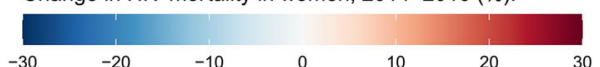

Fig. 3 HIV mortality among men and women in Costa Rica by canton, 2016. HIV mortality per 100,000 by canton in Costa Rica in 2016 among men (a) and women (b). Relative change in HIV mortality between 2014 and 2016 among men (c) and women (d)

to 4.7 [1.8-10] deaths per 100,000 in 2014). Among women, estimated relative change in HIV mortality at the canton level varied from a $199.9 \%$ increase in Río Verde canton, Esmeraldas province (from 3.4 [0.9-10.2] deaths per 100,000 in 2004 to 10.2 [1.7-34.6] deaths per 100,000 in 2014), to essentially no change in Latacunga canton, Cotopaxi province $(0.6[0.3-1.2]$ deaths per 100,000 in 2004 compared to $0.6[0.3-1.3]$ deaths per 100,000 in 2014).

\section{Guatemala}

In Guatemala, estimated national HIV mortality for both sexes combined in 2017 was 4.6 (4.1-5.1) deaths per $100,000(6.8$ [6.2-7.4] deaths for men and 2.8 [2.4-3.1] deaths for women). At the municipality level, estimated HIV mortality for men varied from $0.8(0.2-2.1)$ deaths per 100,000 in the Santa Cruz Barillas municipality, Huehuetenango department to 38.6 (24.5-57.5) deaths per 100,000 in the San José municipality, Escuintla 

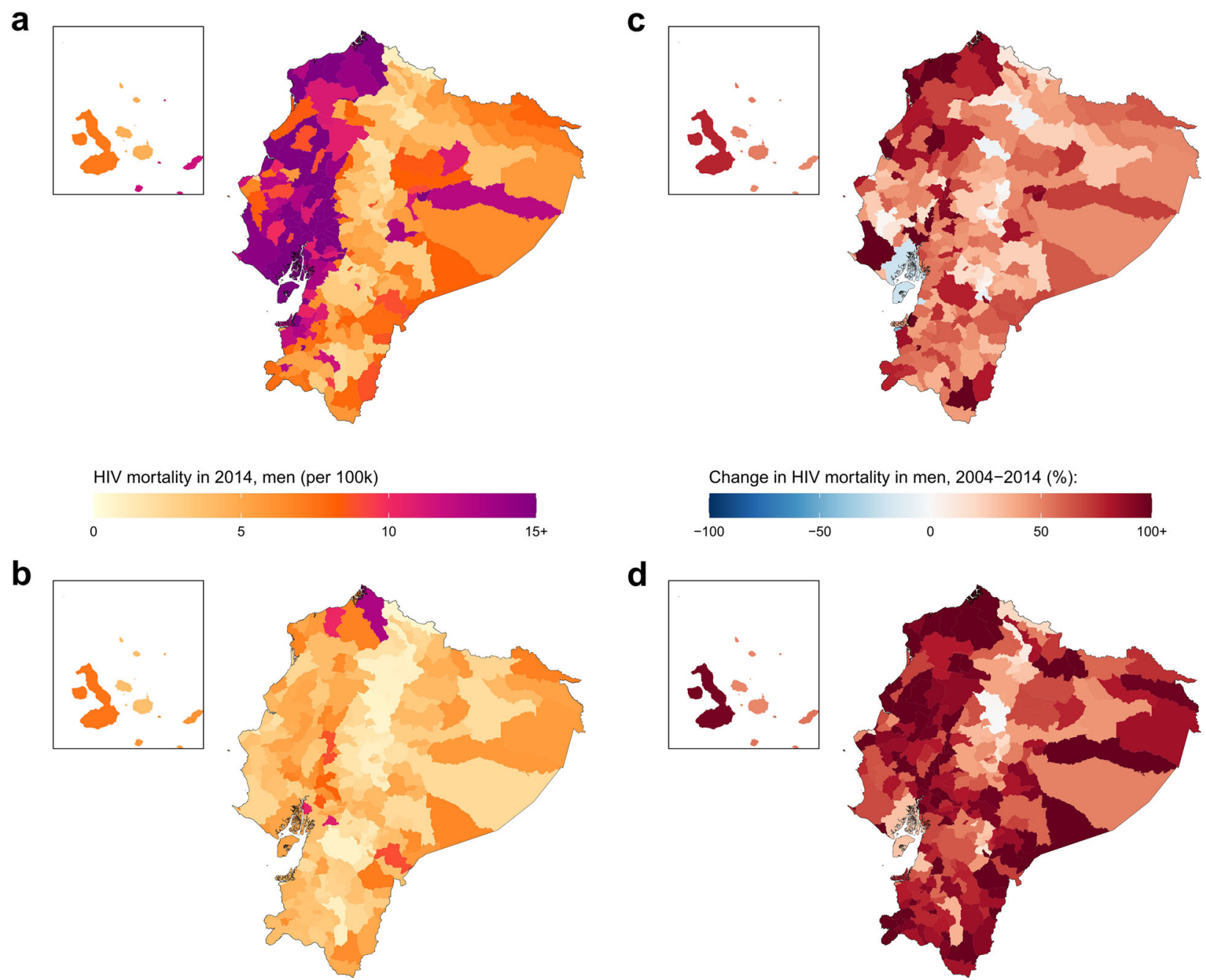

b
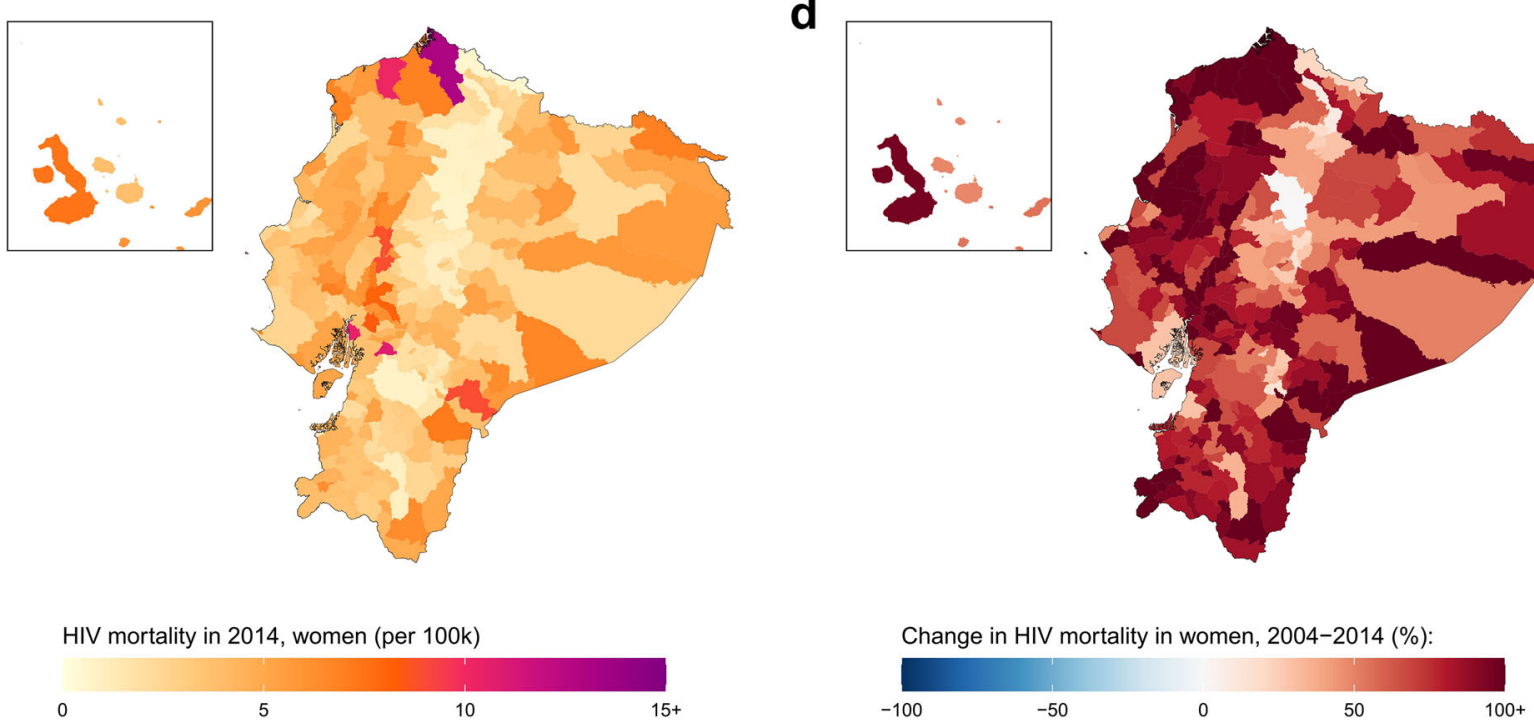

Fig. 4 HIV mortality among men and women in Ecuador by canton, 2014. HIV mortality per 100,000 by canton in Ecuador in 2014 among men (a) and women (b). Relative change in HIV mortality between 2004 and 2014 among men (c) and women (d)

department (Fig. 5 and Additional file 1: Figure S11). For women, estimated HIV mortality ranged from 0.7 ( $0.2-$ 1.5) deaths per 100,000 in the Chiantla municipality, Huehuetenango department to $20.3(12.3-31.8)$ deaths per 100,000 in the San José municipality, Escuintla department. Between 2009 and 2017, estimated national HIV mortality decreased by $36.9 \%$ among men (from 10.8 [9.9-11.7] deaths per 100,000 in 2009) and by $33.5 \%$ among women (from 4.1 [3.7-4.6] deaths per 100, 000 in 2009). Unlike in the other countries considered, estimated HIV mortality decreased in all 340 municipalities for both men and women though only two [0.6\%] municipalities for men and no municipalities for women had a statistically significant decrease in HIV mortality.
Among men, estimated relative change in HIV mortality at the municipality level ranged from a $14.4 \%$ decrease in San José municipality, Escuintla department (from 45 [30.2-65.5] deaths per 100,000 in 2009 to 38.6 [24.557.5 ] deaths per 100,000 in 2017) to a $54 \%$ decrease in Siquinalá municipality, Escuintla department (from 25.4 [14.3-41.3] deaths per 100,000 in 2009 to 11.7 [6.519.7] deaths per 100,000 in 2017). Estimated change in female HIV mortality at the municipality level varied from an $18.5 \%$ decrease in San José municipality, Escuintla department (from 24.9 [15.9-38.6] deaths per 100, 000 in 2009 to 20.3 [12.3-31.8] deaths per 100,000 in 2017) to a $39.9 \%$ decrease in Coatepeque municipality, Quetzaltenango department (from 6.9 [4.4-10.3] deaths 

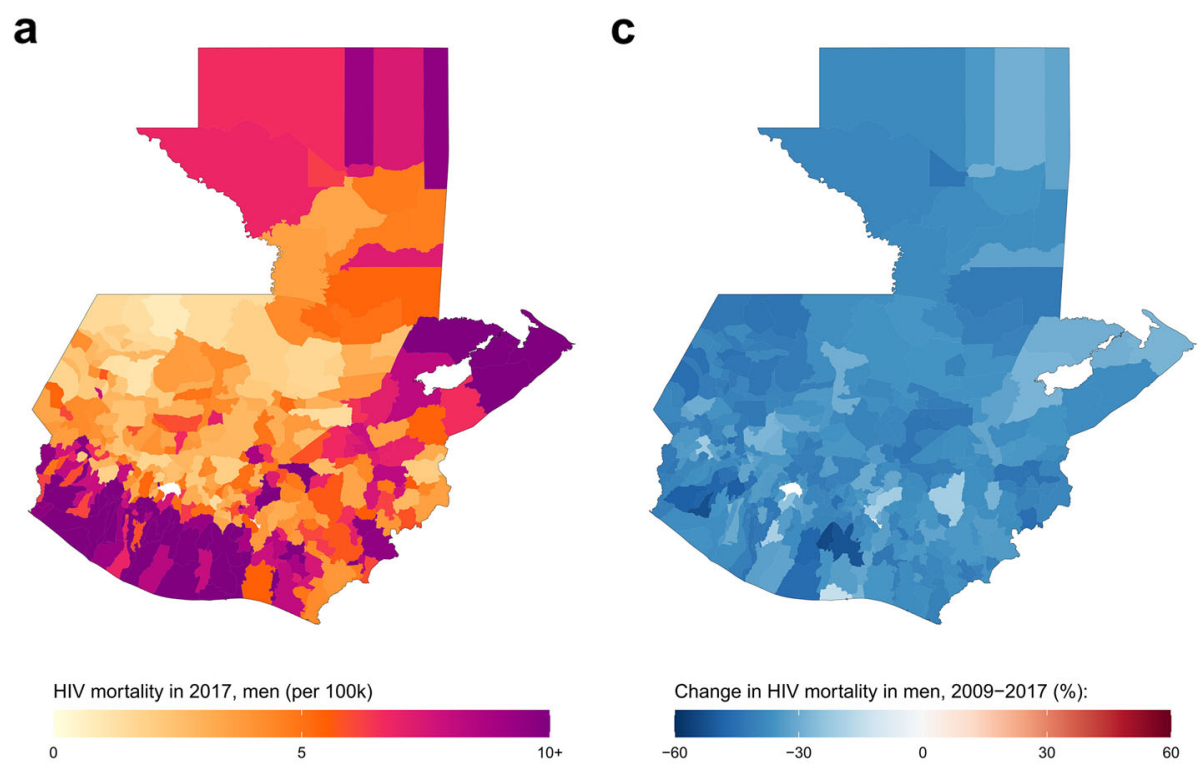

b

d
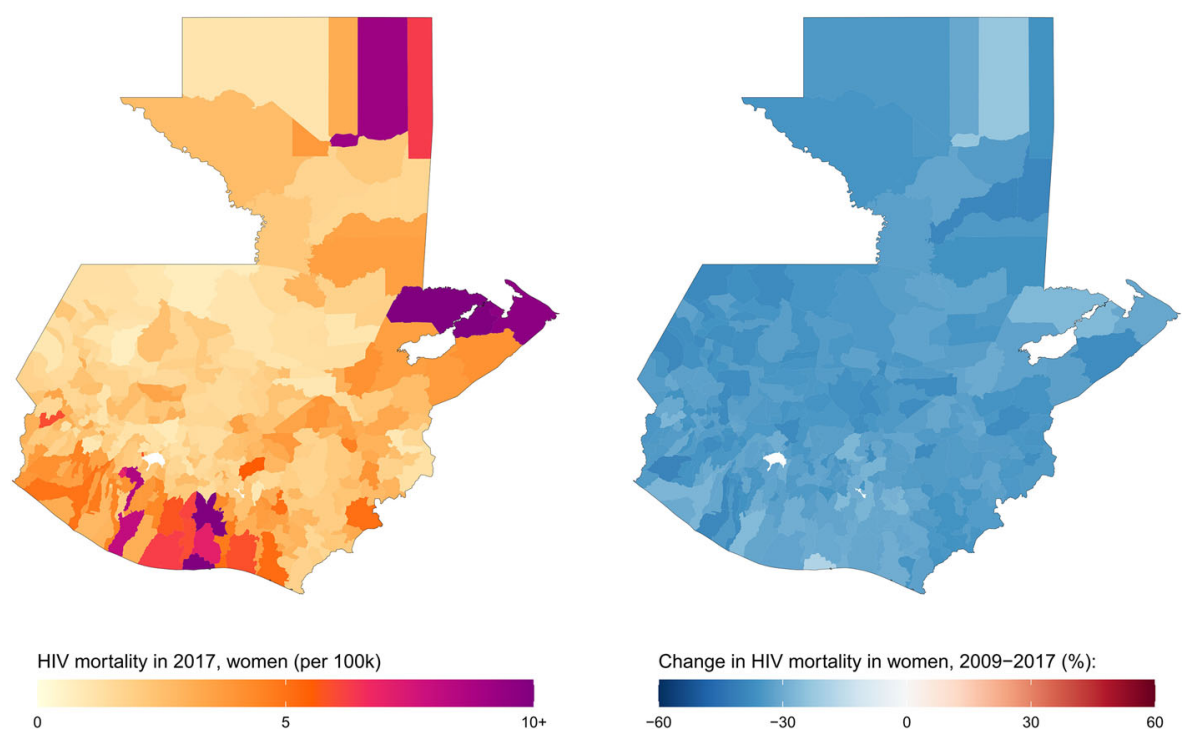

Fig. 5 HIV mortality among men and women in Guatemala by municipality, 2017. HIV mortality per 100,000 by municipality in Guatemala in 2017 among men (a) and women (b). Relative change in HIV mortality between 2009 and 2017 among men (c) and women (d)

per 100,000 in 2009 to 4.1 [2.6-6.6] deaths per 100,000 in 2017).

\section{Mexico}

In Mexico, the estimated national HIV mortality rate for both sexes combined in 2017 was 4.3 (4.2-4.4) deaths per 100,000 (6.9 [6.7-7.1] deaths per 100,000 for men, and 1.9 [1.8-2.0] for women). At the municipality level, estimated HIV mortality for men in 2017 varied over 53fold: from $0.8(0.4-1.3)$ deaths per 100,000 in the San Salvador Atenco municipality, Mexico state to 42.6 (29.1-61.4) deaths in the Tlacotalpan municipality,
Veracruz state (Fig. 6 and Additional file 1: Figure S12). For women, estimated HIV mortality ranged over 47fold from $0.3(0.2-0.6)$ deaths per 100,000 in the Texcoco municipality, Mexico state to 14.2 (8.8-22.0) deaths per 100,000 in the San Juan Cancuc municipality, Chiapas state. Between 2000 and 2017, estimated national HIV mortality decreased by $23.5 \%$ among men (from 9.0 [8.8-9.3] deaths per 100,000 in 2000) and by $5.2 \%$ among women (from 2.0 [1.9-2.1] deaths per 100, 000 in 2000). Estimated male HIV mortality decreased in 2048 (83.3\%) municipalities, and 32 [1.3\%] municipalities had a statistically significant decrease in HIV mortality. 

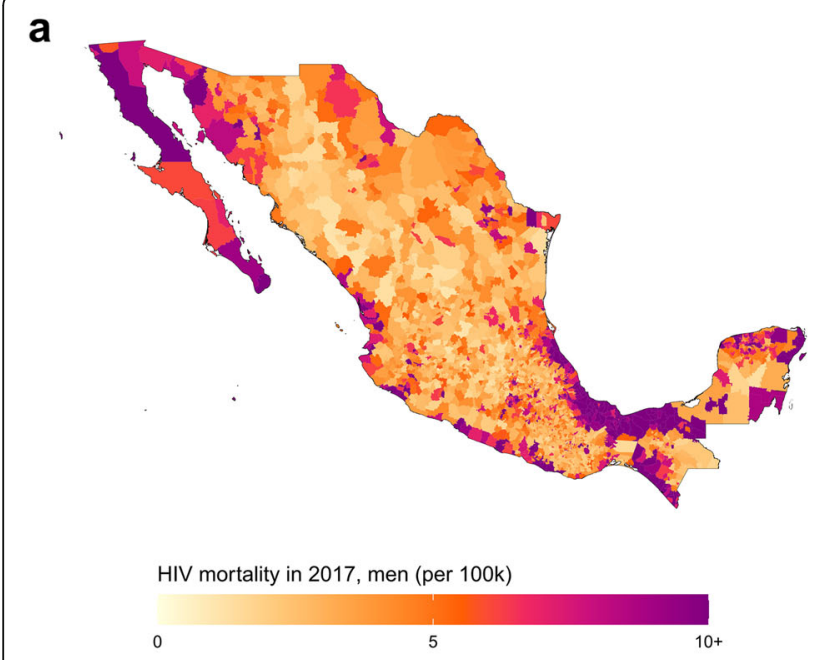

b
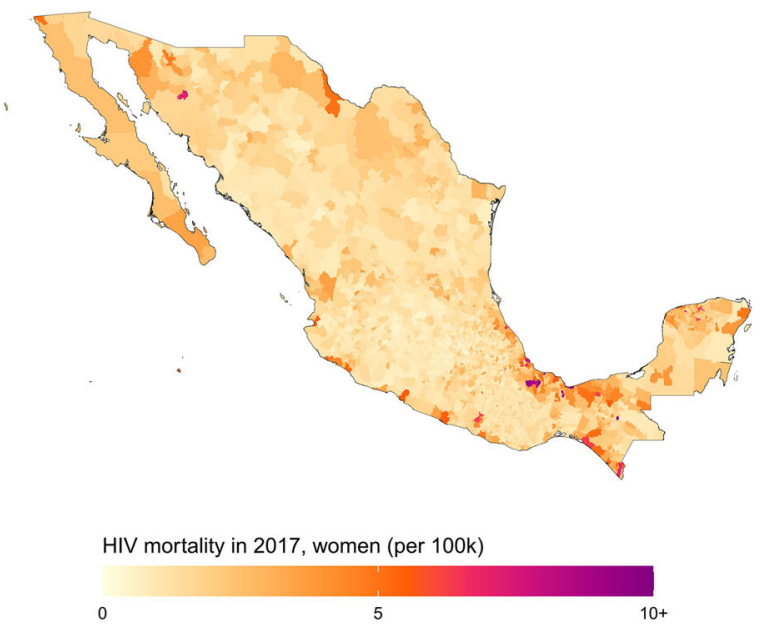

C
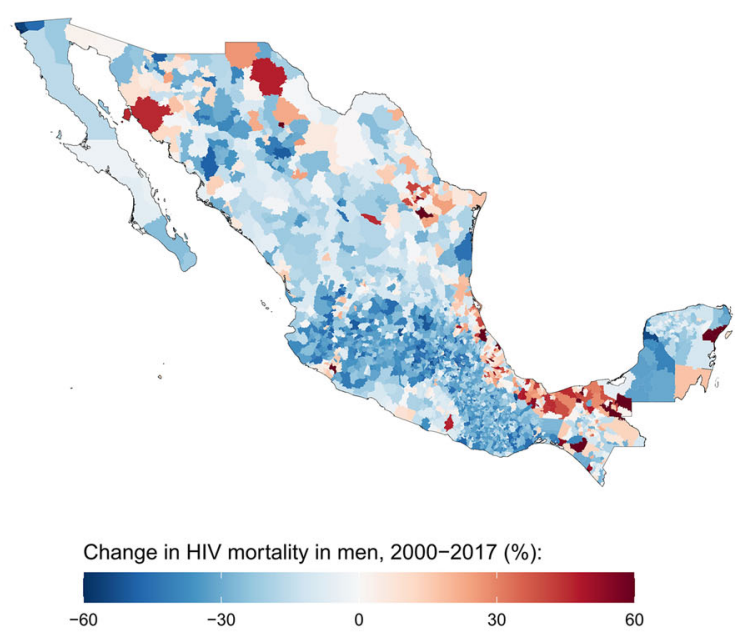

d
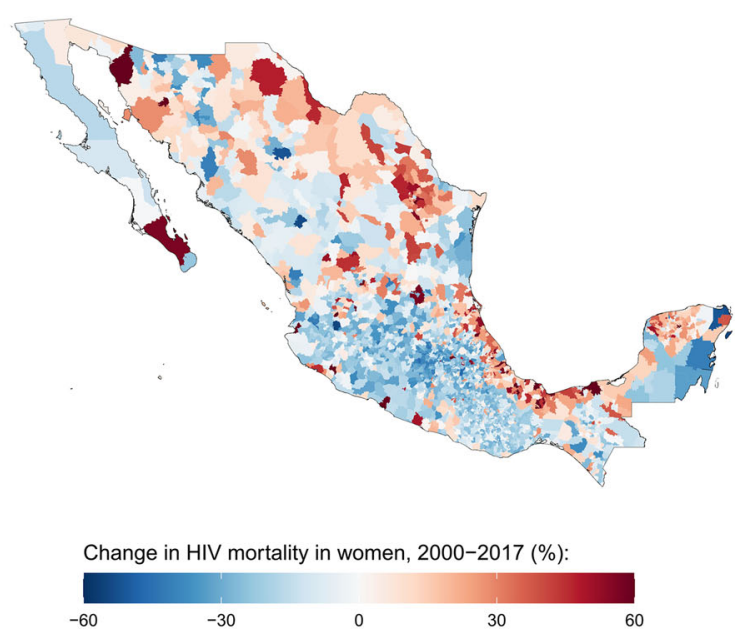

Fig. 6 HIV mortality among men and women in Mexico by municipality, 2017. HIV mortality per 100,000 by municipality in Mexico in 2017 among men (a) and women (b). Relative change in HIV mortality between 2000 and 2017 among men (c) and women (d)

Estimated female HIV mortality decreased in 1683 (68.5\%) municipalities and 4 [0.2\%] municipalities had a significant decrease in HIV mortality. Among men, estimated change in HIV mortality from 2000 to 2017 ranged from a $162.4 \%$ increase in the Solidaridad and Tulum municipalities, Quintana Roo state (from 6.8 [4.9-9.3] deaths per 100,000 in 2000 to 17.9 , [14.5-21.6] deaths per 100,000 in 2017) to a $61.9 \%$ decrease in Playas de Rosarito municipality, Baja California state (from 19.3 [13.6-27.2] deaths per 100,000 in 2000 to 7.4 [5.1-10.2] deaths per 100,000 in 2017). For women, estimated relative change in HIV mortality at the municipality level varied from a $110.3 \%$ increase in Coatzacoalcos municipality, Veracruz state (from 4.5 [3.5-5.8] deaths per 100,000 in 2000 to 9.5 [7.5-11.8] deaths per 100,000 in 2017 ) to a $54.9 \%$ decrease in Zapopan municipality, Jalisco state (from 2.5 [1.9-3.3] deaths per 100,000 in 2000 to 1.1 [0.8-1.5] deaths per 100,000 in 2017).

\section{Concentrated deaths due to HIV}

We estimated that a large proportion of HIV deaths were concentrated in a small number of geographical areas with large populations. In all countries, over half of the HIV deaths were located in less than $10 \%$ of municipalities in the latest year of study (Fig. 7). In Colombia, over half of all HIV deaths in 2017 were located in just $1.2 \%$ (14 of 1122) of municipalities that contain $37.0 \%$ of the total population, and in Guatemala in 2017, over half the HIV deaths were spread out over 9.4\% (32 of 340) of municipalities that contain $33.4 \%$ of the population. Several countries contained single municipalities that contributed a large proportion of national HIV deaths: for example, in Costa Rica in 2016, 45.9\% of all HIV deaths were located in San José canton, San José province, compared to $4.6 \%$ of the total population. Mexico had a greater spread of HIV deaths across municipalities; in 2017 the area with the 
a

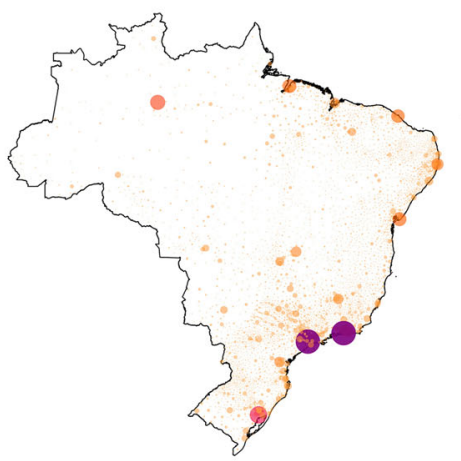

$\begin{array}{ll}\text { HIV Deaths } & 200 \bigcirc 400 \bigcirc 600 \bigcirc 800+ \\ \text { C } & \text { Costa Rica, } 2016\end{array}$

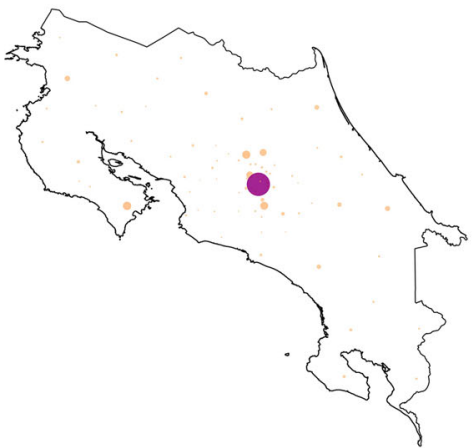

HIV Deaths $\quad 20 \bigcirc 40 \bigcirc 60 \bigcirc 80$

e Guatemala, 2017

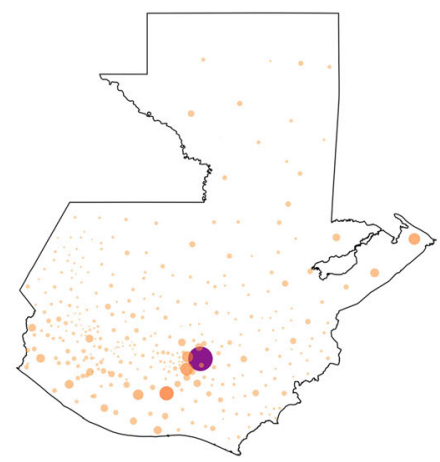

HIV Deaths $\quad 20 \bigcirc 40 \bigcirc 60$ b

Colombia, 2017

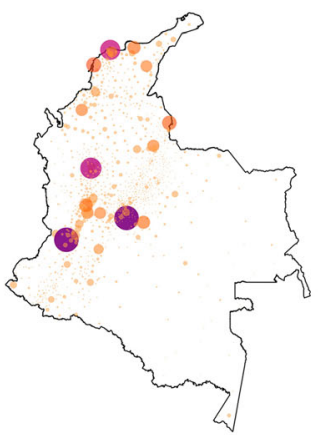

HIV Deaths $\quad 50 \bigcirc 100 \bigcirc 150 \bigcirc 200+$

d

Ecuador, 2014
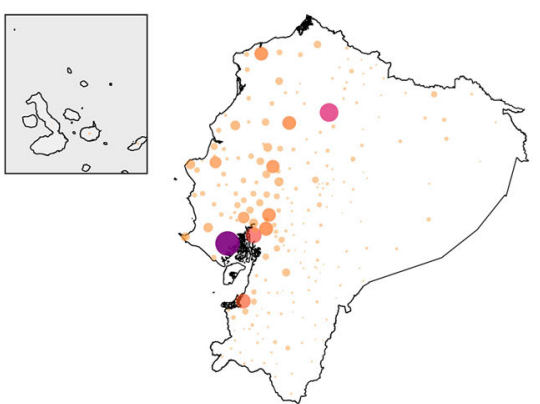

HIV Deaths $\bullet 25 \bigcirc 50 \bigcirc 75 \bigcirc 100+$

f Mexico, 2017

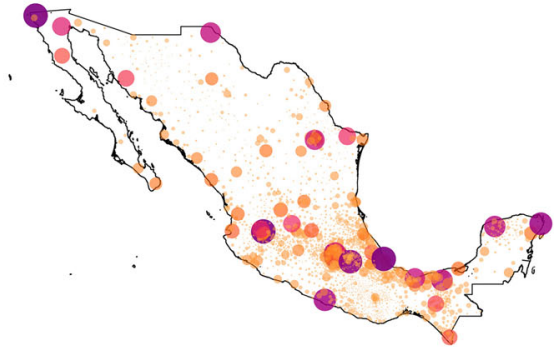

HIV Deaths $\bullet 25 \bigcirc 50 \bigcirc 75$ $100+$

Fig. 7 Number of HIV deaths in latest year of study, by municipality. Estimated number of HIV deaths by municipality in the latest year of study: 2017 in Brazil (a), 2017 in Colombia (b), 2016 in Costa Rica (c), 2014 in Ecuador (d), 2017 in Guatemala (e), 2017 in Mexico (f). Color and size are proportional to estimated HIV deaths

highest proportion of HIV deaths was Tijuana municipality, Baja California state, which amounted to $3.2 \%$ of the total deaths compared to $1.5 \%$ of the population. There was higher HIV mortality among men as compared to women in all countries, ranging from $64.7 \%$ of all deaths concentrated among men in Brazil in 2017, to $77.6 \%$ of all deaths concentrated among men in Mexico in 2017. 


\section{Absolute and relative inequality over time}

Relative inequality - the mortality rate ratio for municipalities in the 90th percentile versus those in the 10th percentile-varied from 5.0 (4.9-5.2) and 4.8 (4.6-5.0) among men and women in Brazil in 2017, to 63.4 (31.6-113.0) and 167.6 (54.6-403.5) among men and women in Costa Rica in 2016 (Fig. 8). The estimated relative geographic inequality in HIV mortality increased in all countries from the first to the last year of study, and this increase was statistically significant in all countries barring Guatemala and Costa Rica. The largest percent increase in relative inequality for each sex over the study period was $49.0 \%$ in Colombian men (from 6.5 [6.0-7.0] in 2000 to 9.6 [8.810.5] in 2017) and 55.9\% in Ecuadorian women (from 4.7 [4.0-5.5] in 2004 to 7.4 [6.1-8.8] in 2014).

Absolute inequality - the difference between the mortality rate in the 90th percentile and the 10th percentile-showed less temporal variation in Brazil, Mexico,

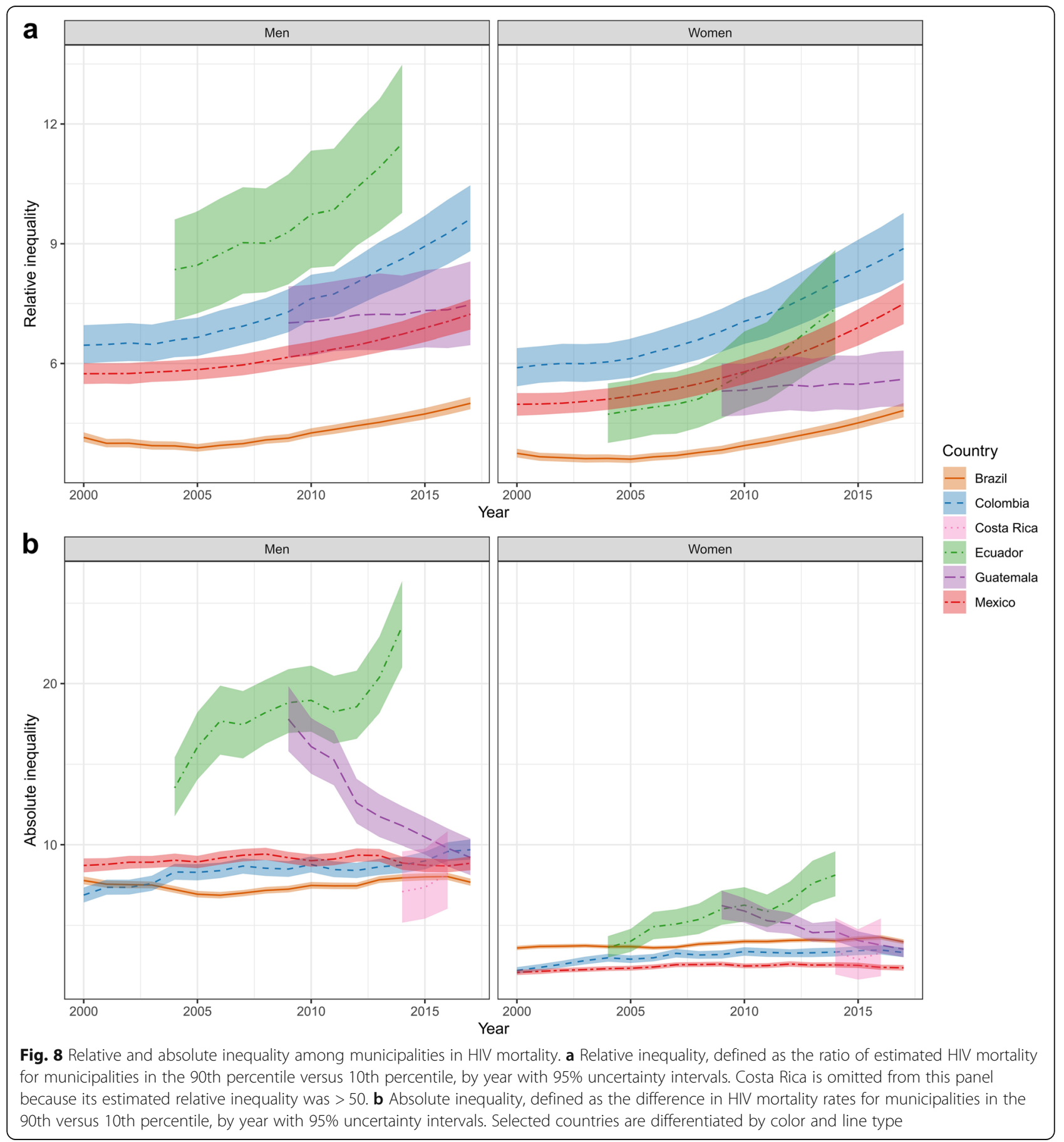


and Costa Rica: the difference in estimated absolute inequality between the first and last year of study was less than 1.5 deaths per 100,000 among both men and women. Male absolute inequality increased in Colombia by $40.9 \%$ (from 6.9 [6.4-7.4] in 2000 to 9.7 [9.1-10.4] in 2017) and in Ecuador by $74.1 \%$ (from 13.5 [11.8-15.4] in 2004 to 23.6 [21.0-26.4] in 2014), while male absolute inequality decreased in Guatemala by $48.2 \%$ (from 17.8 [15.8-19.8] in 2009 to 9.2 [8.1-10.4] in 2017). Female absolute inequality increased in Colombia by $50.8 \%$ (from 2.2 [2.0-2.4] in 2000 to 3.3 [3.0-3.6] in 2017) and Ecuador by $123.9 \%$ (from 3.6 [3.0-4.3] in 2004 to 8.1 [6.8-9.6] in 2014), while female absolute inequality decreased in Guatemala by $43.5 \%$ (from 6.2 [5.4-7.2] in 2009 to 3.5 [3.0-4.1] in 2017). For both men and women, increases in absolute relative inequality in Colombia and Ecuador, as well as decreases in absolute inequality in Guatemala, were statistically significant.

\section{Local disparities in median age group among those who died from HIV}

The estimated median age group among men who died varied substantially at the municipality level in the latest year of study: by 15 years in Brazil, Ecuador, and Mexico, and by 10 years in Colombia, Costa Rica, and Guatemala (Fig. 9). Among women, estimated median age group

\section{a}

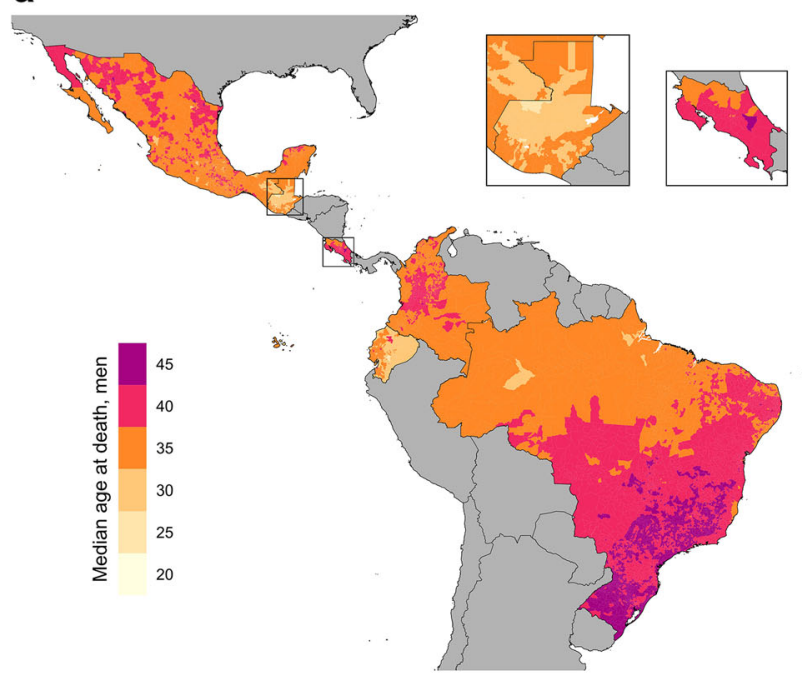

b

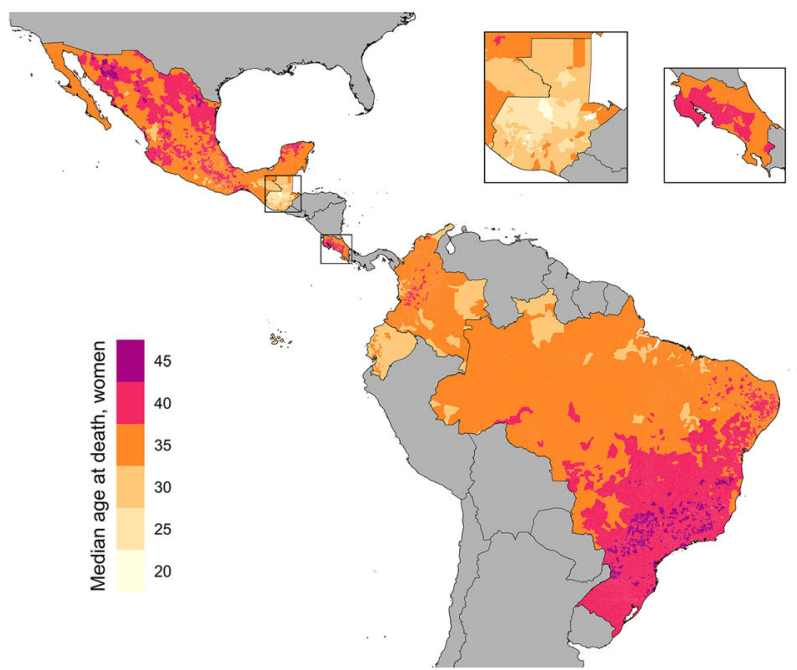

C

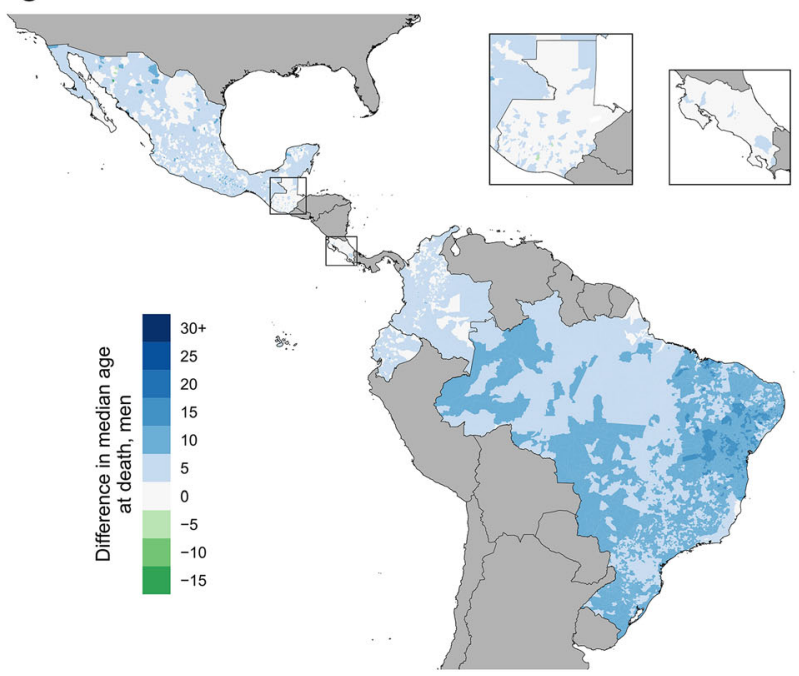

d

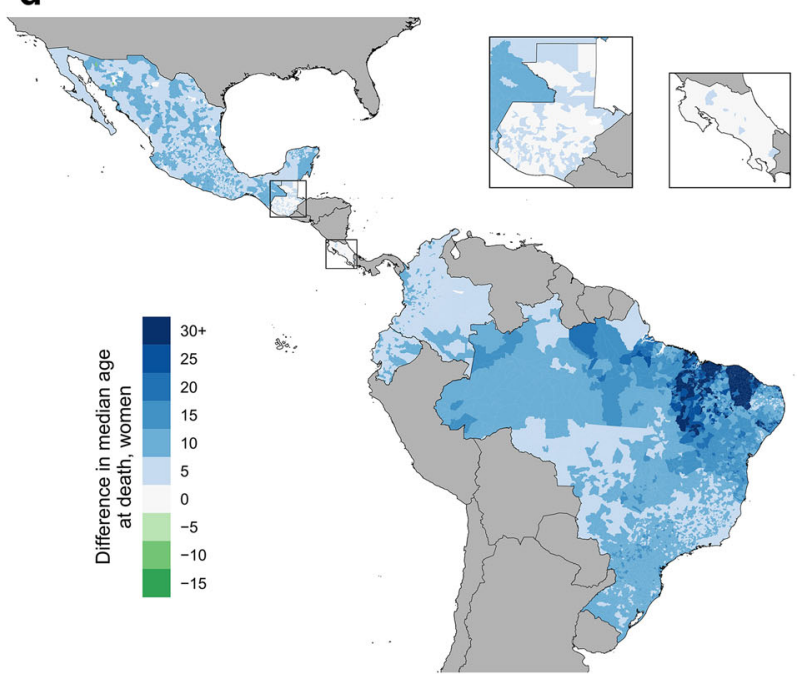

Fig. 9 Estimated median age group among those who died from HIV, by municipality. Estimated median age of death among men (a) and women (b) who died from HIV in the last year of study in selected countries: 2017 in Brazil, Colombia, Guatemala, and Mexico, 2016 in Costa Rica, and 2014 in Ecuador. Estimated difference in median age of death among men (c) and women (d) who died from HIV from first year to last year of study in selected countries (2000-2017 in Brazil, Colombia, and Mexico, 2009-2017 in Guatemala, 2014-2016 in Costa Rica, and 2004-2014 in Ecuador) 
among those who died in the latest year of study varied at the municipality level by 15 years in Brazil, Guatemala, and Mexico, by 10 years in Colombia, and by only 5 years in Costa Rica and Ecuador. Differences in median age group among those who died also shifted over time. In Brazil, the estimated median age group among male decedents rose in $99.6 \%$ of municipalities from 2000 to 2017, while in Guatemala only 21.5\% of municipalities saw an increase in estimated median age group among male decedents from 2009 to 2017. An increase in estimated median age was also observed among women: in Mexico, Ecuador, Colombia, and Brazil, the median age group among female decedents rose in $>97 \%$ of all municipalities in each country. Additional file 1: Figure S16-S21 show estimated HIV mortality by age group and sex for each country in the last year of study.

\section{Discussion}

There are few past analyses that assess HIV mortality in Latin America using VR data at a subnational level, largely due to the statistical challenges of incorporating incomplete VR systems and estimating mortality in areas with small populations and small numbers of deaths. The few analyses that integrate estimates of VR completeness into their modeling framework are often done at the national or state level or are limited to a single country and year [15-18]. In this analysis, we expand on previously described methods [31] that include prior estimates of VR completeness and demonstrate the utility of estimates that combine uncertainty from both incomplete registration systems and from statistical methods designed to leverage information across space, time, and age to inform mortality rates in areas with small numbers of HIV deaths.

Our estimates revealed large-scale spatial heterogeneity in HIV mortality across the six Latin American countries considered in our analysis. We also reveal divergent national trends in HIV mortality in the six countries across the study period, and variable relative change within countries at the municipality level. From the first to the last year of study, HIV mortality decreased among men and women in all countries, with the exception of women in Colombia from 2000 to 2017 and both men and women in Ecuador from 2004 to 2014. Despite the progress in reducing HIV mortality among both sexes at the national level in Brazil, Guatemala, Costa Rica, and Mexico, inequalities in municipality-level HIV mortality persist and relative inequality increased over time in all countries. This analysis highlights uneven progress towards reducing HIV mortality and reaching UNAIDS Fast-Track goals, and emphasizes an alarming trend in Ecuador, where over 95\% of cantons experienced increases in estimated mortality among both sexes from 2004 to 2014 . Nonetheless, it also underlines stories of success: all countries contained municipalities with an estimated decrease in HIV mortality. Further evaluating municipalities with the greatest decreases in HIV mortality within a country may help decision-makers recognize successful strategies that could be implemented in municipalities experiencing increases or slower declines.

There are likely a multitude of factors that contribute to the spatial and temporal patterns in HIV mortality observed in our analysis. Consistent with past analyses, we found higher rates of HIV mortality among men compared to women and slightly different spatial patterns by sex $[15,48,49]$. The consistently elevated levels of mortality among men is likely partially because men who have sex with men (MSM) continue to be one of the populations with the highest prevalence throughout Latin America, and a group that suffers a higher level of stigma and discrimination [48, 50-52]. These trends may also reflect prevalent gender norms [49], unequal access to timely diagnosis and treatment [48], or differences in disease burden from comorbidities between genders [1]. The spatial distribution of high-risk groups possibly also contributes to the HIV epidemic remaining concentrated in large urban centres [6]. For example, one important driver of spatial differences in HIV mortality is prison populations, where HIV transmission is high due to overcrowding, violence, and lack of information on the risk of HIV acquisition [53]. In Brazil, there is evidence of low adherence to antiretroviral therapy (ART) and a higher proportion of primary and secondary resistance among prison populations, which are predominantly male [54]. Municipalities with large prison populations-such as several in the state of Sao Paulo, Brazil-show higher levels of HIV mortality rates and number of deaths due to HIV. Another potential driver of spatial heterogeneity is population migration. Political conflicts and economic hardships across the region, notably in Venezuela and Central America, have fostered waves of migration that can affect HIV prevention, treatment, and care program [55]. Furthermore, difficulties in acquiring HIV treatment and ART shortages spurs regional migration that can differentially impact HIV care and control programs in bordering countries $[55,56]$.

A key driver of temporal trends in HIV mortality is the implementation of ART treatment programs, which have been incorporated to varying degrees in all Latin American countries and generally led to increases in ART coverage [55]. ART treatment is often a central focus of national HIV programs, but differences in priority and ability to commit resources have likely impacted progress in reducing HIV mortality. Access to HIV treatment for people living with HIV is country-dependent and has shifted over time. Brazil was the first middleincome country to offer free ART treatment to people 
living with HIV (PLHIV) in 1996 [57], with Costa Rica following soon after in 1998. Within the ensuing decade, Mexico [58] and Colombia [59] adopted similar policies of universal treatment, which may have contributed to the observed reduction in national HIV mortality as these programs matured. In recent years, Guatemala has also expanded ART treatment options through joint support from the national government and The Global Fund to Fight AIDS, Tuberculosis, and Malaria [60]. Given the concentration of HIV in urban high-risk groups, many national programs-such as those in Costa Rica and Guatemala-have emphasized a combination prevention strategy that focus on HIV testing, STI diagnosis, and linkage to care in vulnerable populations [61]. In Ecuador, the only country in our analysis where we estimated increases in national HIV mortality among men and women over the study period, there has historically been a paucity of information available on research findings concerning HIV/AIDS burden [62]. Notably, our analysis in Ecuador only extends to 2014 and patterns of HIV mortality may have changed with the recent emphasis on community testing and treatment in metropolitan areas [63]. While all countries have expanded access to treatment and documented increases in ART coverage, albeit at different time periods, persistent disparities in access to quality health services and adherence to ART remain $[6,64,65]$ and may contribute to differences in HIV mortality declines observed in this analysis. Further, in all countries in our analysis, communities with socioeconomic and health disadvantages-such as indigenous communities, sex workers, and transgender populations-often have unequal access to treatment and are an emerging or establish public health priority $[66,67]$.

Our analysis also revealed substantial variation in median age group among those who died from HIV at the municipality level, and an increase over time in median age group among those who died of HIV. These results agree with past research that demonstrates an accelerating growth in the number of people living with HIV that are above 50 years of age $[68,69]$. Several countries, notably Brazil and Mexico, contained municipalities with a 15 -year difference in median age groups among men and women who died from HIV in the latest year of study. While attributing these trends to specific drivers is outside the scope of this analysis, there are several factors that could influence an increase in median age of death, including changes in HIV incidence in specific age groups [70], increases in life expectancy among people living with HIV [70], access to ART [71], migration, and the age distribution of the population across the study period.

This analysis provides novel subnational estimates of HIV mortality that convey important information to policymakers and could inform future action. Knowledge of local differences in HIV mortality can help guide scale-up of ART where mortality might reflect suboptimal coverage. Our estimates highlight how deaths due to HIV are concentrated in a low proportion of municipalities. In the longer term, HIV mortality measures could be used to highlight areas that might benefit from programmatic interventions that target HIV prevention such as pre-exposure prophylaxis (PrEP). As countries in Latin America consider expanding access to PrEP, studies have demonstrated that prioritization of PrEP to those at highest risk could save money and lives $[72,73]$. Cost-effective interventions are especially important in Guatemala, Ecuador, Colombia, and Costa Rica, where HIV programs depend on donor funding [74]. Furthermore, subnational differences in HIV burden have already been used to develop localized strategies for HIV prevention and elimination in subSaharan Africa [75, 76].

\section{Limitations}

This analysis is subject to a number of limitations. First, the VR data that inform our estimates are subject to misclassification biases. HIV is generally under-reported as a cause of death [23]. While the HIV correction methodology from the GBD employed in this analysis corrects for biases in HIV deaths classified to other underlying causes of death, there may be additional country-specific biases not addressed by our methodological approach. While we matched decedents to their municipality of residence as provided by the VR mortality databases, these data could overrepresent urban areas with larger health care facilities where individuals may have died after seeking treatment. It is possible that in some cases these individuals may have been mistakenly recorded as residing in that municipality if their municipality of residence was not available. Second, our method for correcting for incomplete VR across space and time makes several crucial assumptions. Our analysis incorporates estimates of VR completeness for children under 15 and adults 15 and over based on previous analyses, but VR completeness may vary within these age ranges. Further, we use the geographic variation in completeness identified by comparing reported under- 5 all-cause deaths to previous estimates, and the variation in under-5 mortality may not be comparable to patterns in adult VR incompleteness. Additionally, variation in all-cause completeness may differ from patterns in HIVspecific VR completeness. Third, we use population estimates from WorldPop in this analysis that are subject to error, especially in sparsely populated areas. While WorldPop estimates include census data as inputs [77], depending on timing and data accessibility, estimates may differ from the underlying census measures and 
may not utilize the most recent census or the most detailed tabulations. Fourth, population migration in response to political conflict or economic instability in the region, including Central America and Venezuela, may not be properly captured in WorldPop estimates or recorded in vital registration systems. Fifth, our analysis is subject to large uncertainty. This reflects uncertainty both due to the small number of HIV deaths at the municipality level and the need to estimate completeness. While we believe this method better captures major sources of uncertainty, care must be taken when interpreting results. Sixth, we use custom shapefiles that are matched to country-level administrative subdivisions, and differences in administrative divisions between GAUL [35] or the Humanitarian Data Exchange [36] and an individual country's designation of administrative areas may affect the accuracy of results, especially in our estimates of the number of HIV deaths by municipality. Seventh, our small area estimation models smooth over space and time by making assumptions about the temporal and spatial structure of HIV mortality that may not always hold. Eighth, VR data availability varied across the countries selected in our analysis, and comparison between temporal trends in HIV mortality may be difficult to assess for countries with different years of data availability. Finally, it is difficult to directly assess violations of our modeling assumptions or quality issues in the underlying data sources given that VR completeness cannot be verified. Nonetheless, comparisons to GBD national estimates (Additional file 1: Figure S4-S9) provide reassurance in overall country trends.

\section{Future directions}

There is considerable opportunity to expand this analysis. First, access to VR data over more years of study, or in neighboring countries in Latin America, could provide valuable benchmarks for more direct comparisons and allow additional information across space and time to potentially improve our models. Our current study uses four available covariates that serve as proxies for urbanization and development, but in the future, availability of other drivers of HIV mortality at the municipality level such as socioeconomic status, healthcare infrastructure, high-risk group concentration, and ART treatment availability could improve our estimates. Further, the technique we used to include uncertainty and information on subnational VR completeness could be extended to other countries where VR systems are not complete. Finally, this small area estimation framework could be used to estimate all-cause and cause-specific mortality due to other causes at local levels in the six modeled Latin American countries.

\section{Conclusion}

Our analysis finds large-scale variation in HIV mortality among municipalities in six Latin American countries, both in the latest year of study as well as over the entire study period. Our estimates demonstrate the need to assess HIV burden at a granular geographic scale in Latin America, given that the HIV epidemic is concentrated in high-risk groups and select urban areas. The methods developed in this analysis provide a framework for incorporating prior information on VR completeness into subnational estimates of HIV burden. This analysis could be used to identify areas that have successfully reduced HIV mortality and areas of high HIV burden, as well as to inform the rollout of preventive interventions that are required to help countries progress towards achieving UNAIDS targets and advance health equity.

\section{Supplementary Information}

The online version contains supplementary material available at https://doi. org/10.1186/s12916-020-01876-4.

\begin{abstract}
Additional file 1:. Supplemental methods, GATHER checklist, Supplemental Figure S1-S21, and Supplemental Tables S1-S4. Figure S1. Analytical process overview. Figure S2. Analytical process for VR data. Figure S3. Analytical process overview for VR completeness priors. Figure S4. Model alignment with GBD, Brazil. Figure S5. Model alignment with GBD, Colombia. Figure S6. Model alignment with GBD, Costa Rica. Figure S7. Model alignment with GBD, Ecuador. Figure S8. Model alignment with GBD, Guatemala. Figure S9. Model alignment with GBD, Mexico. Figure S10. Mean and uncertainty in estimated HIV mortality in Brazil, 2017. Figure S11. Mean and uncertainty in estimated HIV mortality in Colombia, 2017. Figure S12. Mean and uncertainty in estimated HIV mortality in Costa Rica, 2016. Figure S13. Mean and uncertainty in estimated HIV mortality in Ecuador, 2014. Figure S14. Mean and uncertainty in estimated HIV mortality in Guatemala, 2017. Figure S15. Mean and uncertainty in estimated HIV mortality in Mexico, 2017. Figure S16. Estimated HIV mortality in Brazil by age group, 2017. Figure S17. Estimated HIV mortality in Colombia by age group, 2017. Figure S18. Estimated HIV mortality in Costa Rica by age group, 2016. Figure S19. Estimated HIV mortality in Ecuador by age group, 2014. Figure S20. Estimated HIV mortality in Guatemala by age group, 2017. Figure S21. Estimated HIV mortality in Mexico by age group, 2017. Table S1: Merged municipalities by country to form stable geographical units. Table S2: Vital Registration data. Table S3: Covariate data sources. Table S4: National HIV mortality rates among men and womenfwil.
\end{abstract}

\section{Acknowledgements}

Local Burden of Disease HIV Collaborators:

Michael A Cork', Nathaniel J Henry',2, Stefanie Watson', Andrew J Croneberger', Mathew Baumann', Ian D Letourneau', Mingyou Yang', Audrey L Serfes ${ }^{1}$, Jaffar Abbas ${ }^{3}$, Nooshin Abbasi $^{4}$, Hedayat Abbastabar ${ }^{5}$, Lucas G Abreu ${ }^{6}$, Eman Abu-Gharbieh", Basavaprabhu Achappa ${ }^{8}$, Maryam Adabi ${ }^{9}$, Tadele G Adal ${ }^{10}$, Adeyinka E Adegbosin ${ }^{11}$, Victor Adekanmbi ${ }^{12}$, Olatunji O Adetokunboh ${ }^{13,14}$, Marcela Agudelo-Botero ${ }^{15}$, Bright O Ahinkorah ${ }^{16}$, Keivan Ahmadi $^{17}$, Muktar B Ahmed ${ }^{18,19}$, Robert K Alhassan ${ }^{20}$, Vahid Alipour ${ }^{21,22}$, Amir Almasi-Hashiani ${ }^{23}$, Nelson Alvis-Guzman ${ }^{24,25}$, Robert Ancuceanu ${ }^{26}$, Tudorel Andrei $^{27}$, Davood Anvari ${ }^{28,29}$, Muhammad Aqeel ${ }^{30}$, Jalal Arabloo ${ }^{21}$, Olatunde Aremu $^{31}$, Malke Asaad ${ }^{32}$, Desta D Atnafu' ${ }^{33}$, Alok Atreya ${ }^{34}$, Beatriz Paulina Ayala Quintanilla ${ }^{35}$, Samad Azari ${ }^{21}$, Darshan B B ${ }^{36}$, Atif A Baig ${ }^{37}$, Maciej Banach $^{38,39}$, Simachew A Bante ${ }^{40}$, Miguel A Barboza ${ }^{41,42}$, Sanjay Basu ${ }^{43,44}$, Neeraj Bedi ${ }^{45,46}$, Diana F Bejarano Ramirez ${ }^{47,48}$, Isabela M Bensenor ${ }^{49}$, Fentahun Y Beyene ${ }^{40}$, Yihienew M Bezabih ${ }^{50,51}$, Akshaya S Bhagavathula ${ }^{52,53}$, Nikha Bhardwaj ${ }^{54}$, Pankaj Bhardwaj ${ }^{55,56}$, Krittika Bhattacharyya ${ }^{57,58}$, Zulfiqar A Bhutta $^{59,60}$, Ali Bijani ${ }^{61}$, Sait M Birlik ${ }^{62,63}$, Zebenay W Bitew ${ }^{64}$, Somayeh 
Bohlouli ${ }^{65}$, Archith Boloor ${ }^{8}$, Andre R Brunoni ${ }^{49,66}$, Zahid A Butt ${ }^{67,68}$, Rosario Cárdenas $^{69}$, Felix Carvalho ${ }^{70}$, Joao Mauricio Castaldelli-Maia ${ }^{66}$, Carlos A Castañeda-Orjuela ${ }^{71,72}$, Jaykaran Charan ${ }^{73}$, Souranshu Chatterjee ${ }^{74}$, Vijay Kumar Chattu $^{75}$, Soosanna Kumary Chattu ${ }^{76}$, Mohiuddin Ahsanul Kabir Chowdhury ${ }^{77,78}$, Devasahayam J Christopher ${ }^{79}$, Dinh-Toi Chu ${ }^{80}$, Aubrey J Cook', Natalie M Cormier', Saad M A Dahlawi ${ }^{81}$, Farah Daoud', Claudio A DávilaCervantes $^{82}$, Nicole Davis Weaver ${ }^{1}$, Fernando P De la Hoz ${ }^{83}$, Feleke M Demeke $^{84}$, Edgar Denova-Gutiérrez ${ }^{85}$, Kebede Deribe ${ }^{86,87}$, Keshab Deuba ${ }^{88,89}$, Samath D Dharmaratne ${ }^{1,90,91}$, Govinda P Dhungana ${ }^{92}$, Daniel Diaz ${ }^{93,94}$, Shirin Djalalinia $^{95}$, Andre R Duraes ${ }^{96,97}$, Arielle W Eagan ${ }^{98,99}$, Lucas Earl ${ }^{1}$, Andem Effiong ${ }^{100}$, Maysaa El Sayed Zaki ${ }^{101}$, Maha El Tantawi ${ }^{102}$, Rajesh Elayedath ${ }^{103}$, Shaimaa I El-Jaafary ${ }^{104}$, Emerito Jose A Faraon ${ }^{105}$, Andre Faro ${ }^{106}$, Nazir Fattahi $^{107}$, Nelsensius K Fauk ${ }^{108}$, Eduarda Fernandes ${ }^{109}$, Irina Filip ${ }^{110,111}$, Florian Fischer $^{112}$, Nataliya A Foigt ${ }^{113}$, Masoud Foroutan ${ }^{114}$, Takeshi Fukumoto ${ }^{115}$, Mohamed M Gad ${ }^{116,117}$, Tesfay B B Gebremariam ${ }^{118}$, Ketema B Gebremedhin $^{119}$, Gebreamlak G Gebremeskel ${ }^{120,121}$, Hailay A Gesesew ${ }^{108,122}$, Keyghobad Ghadiri $^{123,124}$, Ahmad Ghashghaee ${ }^{21,125}$, Syed Amir Gilani ${ }^{126}$, Mahaveer Golechha ${ }^{127}$, Ugo Gori ${ }^{128}$, Alessandra C Goulart ${ }^{49,129}$, Bárbara N G Goulart ${ }^{130}$, Harish C Gugnani ${ }^{131,132}$, Mark D C Guimaraes ${ }^{133}$, Rafael A Guimarães ${ }^{134}$, Yuming Guo ${ }^{135,136}$, Rahul Gupta ${ }^{137,138}$, Emily Haeuser', Mohammad Rifat Haider ${ }^{139}$, Teklehaimanot G Haile ${ }^{120}$, Arvin Haj-Mirzaian ${ }^{140,141}$, Arya Haj-Mirzaian ${ }^{142}$, Asif Hanif $^{143}$, Arief Hargono ${ }^{144}$, Ninuk Hariyani ${ }^{145,146}$, Soheil Hassanipour ${ }^{147,148}$, Hadi Hassankhani ${ }^{149}$, Khezar Hayat ${ }^{150,151}$, Claudiu Herteliu ${ }^{27,152}$, Hung Chák $\mathrm{Ho}^{153}$, Ramesh Holla ${ }^{154}$, Mehdi Hosseinzadeh ${ }^{155,156}$, Mowafa Househ ${ }^{157}$, BingFang Hwang ${ }^{158}$, Charles U Ibeneme ${ }^{159,160}$, Segun E Ibitoye ${ }^{161}$, Olayinka S Ilesanmi ${ }^{162,163}$, Milena D $\|_{i c^{164}}$, Irena M Ilic ${ }^{165}$, Usman Iqbal ${ }^{166}$, Deepa Jahagirdar' $^{1}$, Vardhmaan Jain ${ }^{167}$, Mihajlo Jakovljevic ${ }^{168,169}$, Ravi P Jha ${ }^{170,171}$, Kimberly B Johnson', Nitin Joseph ${ }^{36}$, Farahnaz Joukar ${ }^{147,148}$, Leila R Kalankesh ${ }^{172}$, Rohol lah Kalhor ${ }^{173,174}$, Tanuj Kanchan ${ }^{175}$, Behzad Karami Matin ${ }^{107}$, André Karch ${ }^{176}$, Salah Eddin Karimi ${ }^{177}$, Getinet Kassahun ${ }^{178}$, Gbenga A Kayode ${ }^{179,180}$, Ali Kazemi Karyani ${ }^{107}$, Maryam Keramati ${ }^{181}$, Nauman Khalid ${ }^{182}$, Ejaz A Khan ${ }^{183}$, Gulfaraz Khan ${ }^{184}$, Md Nuruzzaman N Khan ${ }^{185,186}$, Khaled Khatab ${ }^{187,188}$, Neda Kianipour ${ }^{189}$, Yun Jin Kim ${ }^{190}$, Sezer Kisa ${ }^{191}$, Adnan Kisa ${ }^{192,193}$, Soewarta Kosen ${ }^{194}$, Sindhura Lakshmi Koulmane Laxminarayana ${ }^{195}$, Ai Koyanagi ${ }^{196}$ Kewal Krishan ${ }^{197}$, Barthelemy Kuate Defo ${ }^{198,199}$, Ricardo S Kuchenbecker ${ }^{130,200}$, Vaman Kulkarni ${ }^{36}$, Nithin Kumar ${ }^{36}$, Manasi Kumar ${ }^{201,202}$, Om P Kurmi ${ }^{203,204}$, Dian Kusuma ${ }^{205,206}$, Carlo La Vecchia ${ }^{207}$, Dharmesh K Lal ${ }^{208}$, Iván Landires ${ }^{209}$, Savita Lasrado ${ }^{210}$, Paul H Lee ${ }^{211}$, Kate E LeGrand', Bingyu Li '212, Shanshan $\mathrm{Li}^{213}$, Xuefeng Liu' ${ }^{214}$, Hawraz I M. Amin ${ }^{215,216}$, Daiane B Machado ${ }^{217,218}$, Deepak Madi ${ }^{8}$, Carlos Magis-Rodriguez ${ }^{219}$, Deborah C Malta ${ }^{220}$, Mohammad Ali Mansournia ${ }^{221}$, Md Dilshad Manzar ${ }^{222}$, Carlos A Marrugo Arnedo ${ }^{24,223}$, Francisco R Martins-Melo 224 , Seyedeh Zahra Masoumi ${ }^{225}$, Benjamin K Mayala, ${ }^{1,226}$ Carlo E Medina-Solís ${ }^{227}$, Ziad A Memish 228,229, Walter Mendoza ${ }^{230}$, Ritesh G Menezes ${ }^{231}$, Tomislav Mestrovic ${ }^{232,233}$, Andreea Mirica ${ }^{234}$, Babak Moazen ${ }^{235,236}$, Yousef Mohammad ${ }^{237}$, Naser Mohammad Gholi Mezerji ${ }^{238}$, Abdollah Mohammadian-Hafshejani $i^{239}$, Reza Mohammadpourhodki ${ }^{240}$, Shafiu Mohammed $^{235,241}$, Ali H Mokdad ${ }^{1,91}$, Mohammad Ali Moni ${ }^{242}$, Masoud Moradi ${ }^{107}$, Yousef Moradi ${ }^{243}$, Rahmatollah Moradzadeh ${ }^{23}$, Paula Moraga ${ }^{244}$, Amin Mousavi Khaneghah245, Ghulam Mustafa ${ }^{246,247}$, Lillian Mwanri ${ }^{208}$, Ravishankar Nagaraja248, Ahamarshan J Nagarajan 249,250, Mukhammad David Naimzada $^{251,252}$, Bruno R Nascimento ${ }^{253,254}$, Dr M Naveed ${ }^{255}$, Vinod C Nayak ${ }^{256}$ Javad Nazari ${ }^{257}$, Hadush Negash ${ }^{258}$, Ionut Negoi ${ }^{259,260}$, 'Samata Nepal ${ }^{261}$, Georges Nguefack-Tsague ${ }^{262}$, Cuong T Nguyen ${ }^{263}$, Huong L T Nguyen ${ }^{263}$, Rajan Nikbakhsh ${ }^{141}$, Jean Jacques Noubiap ${ }^{264}$, Virginia Nunez-Samudio ${ }^{265,266}$, Bogdan Oancea 267', Felix A Ogbo ${ }^{268}$, Andrew T Olagunju ${ }^{269,270}$, Nikita Otstavnov ${ }^{251}$, Mahesh P A ${ }^{271}$, Jagadish Rao Padubidri ${ }^{256}$, Seithikurippu R PandiPerumal ${ }^{272}$, Ana M Pardo-Montaño ${ }^{273}$, Urvish K Patel ${ }^{274}$, Shrikant Pawar ${ }^{275}$, Emmanuel K Peprah ${ }^{276}$, Alexandre Pereira ${ }^{277,278}$, Samantha Perkins', Julia 'M Pescarini ${ }^{279}$, Khem N Pokhrel ${ }^{280}$, Maarten J Postma ${ }^{281,282}$, Faheem H Pottoo $^{283}$, Sergio I Prada 284,285 , Liliana Preotescu ${ }^{286,287}$, Dimas R A Pribadi ${ }^{288}$, Amir Radfar ${ }^{289}$, Fakher Rahim ${ }^{290,291}$, Mohammad Hifz Ur Rahman ${ }^{292}$, Amir Masoud Rahmani ${ }^{155,293}$, Kiana Ramezanzadeh ${ }^{294}$, Juwel Rana ${ }^{295,296}$, 'Chhabi L Ranabhat ${ }^{297,298}$, Sowmya J Rao ${ }^{299}$, Priya Rathi ${ }^{154}$, Salman Rawaf ${ }^{300,301}$, David L Rawaf ${ }^{302,303}$, Reza Rawassizadeh ${ }^{304}$, Vishnu Renjith ${ }^{305}$, Nima Rezaei ${ }^{306,307}$, Aziz Rezapour $^{21}$, Ana Isabel Ribeiro ${ }^{308}$, Leonardo Roever ${ }^{309}$, Enrico Rubagotti ${ }^{310}$, Susan F Rumisha ${ }^{311,312}$, Godfrey M Rwegerera ${ }^{313}$, Rajesh Sagar ${ }^{314}$, S. Mohammad Sajadi ${ }^{315,316}$, Marwa R Salem ${ }^{317}$, Abdallah M Samy ${ }^{318}$, Rodrigo Sarmiento-Suárez ${ }^{319,320}$, Brijesh Sathian ${ }^{321,322}$, Lauren E Schaeffer', Ione J C Schneider ${ }^{323}$, Abdul-Aziz Seidu ${ }^{324,325}$, Feng Sha ${ }^{326}$, Masood A Shaikh ${ }^{327}$, Kiomars Sharafi ${ }^{107}$, Aziz Sheikh ${ }^{328,329}$, Kenji Shibuya ${ }^{330}{ }^{2}$, Jae II Shin ${ }^{331}$, Diego A S Silva $^{332}$, Jasvinder A Singh ${ }^{333,334}$, Valentin Y Skryabin ${ }^{335}$, Anna A Skryabina ${ }^{336}$,
Amber Sligar ${ }^{1}$, Amin Soheili ${ }^{337}$, Krista M Steuben ${ }^{1}$, Mu'awiyyah B Sufiyan $^{338}$, Eyayou G Tadesse ${ }^{339}$, Ayenew K T Tesema ${ }^{340}$, Fisaha H Tesfay ${ }^{341,342}$, Rekha' Thapar $^{36}$, Robert L Thompson', Marcos R Tovani-Palone ${ }^{343,344}$, Bach X Tran ${ }^{345}$ Gebiyaw W Tsegaye ${ }^{346}$, Chukwuma D Umeokonkwo ${ }^{347}$, Bhaskaran Unnikrishnan $^{154}$, Yasser Vasseghian ${ }^{155}$, Francesco S Violante ${ }^{348,349}$, Bay Vo ${ }^{350}$, Giang T $\mathrm{Vu}^{351}$, Yasir Waheed ${ }^{352}$, Yuan-Pang Wang ${ }^{66}$, Yanzhong Wang ${ }^{353}$, Paul Ward ${ }^{108}$ Fissaha T Welay ${ }^{354}$, Ronny Westerman ${ }^{355}$, Nuwan D Wickramasinghe ${ }^{356}$, Sanni Yaya ${ }^{357,358}$, Paul Yip ${ }^{359,360}$, Naohiro Yonemoto ${ }^{361,362}$, Chuanhua Yu ${ }^{363,364}$, Deniz Yuce ${ }^{365}$, Hasan Yusefzadeh ${ }^{366}$, Maryam Zamaniann ${ }^{23}$, Mikhail S Zastrozhin $^{367,368}$, Zhi-Jiang Zhang ${ }^{369}$, Yunquan Zhang ${ }^{370,371}$, Arash Ziapour ${ }^{372}$, Simon I Hay ${ }^{1,91 *}$, Laura Dwyer-Lindgren ${ }^{1,91 *}$.

*Joint senior authors

1. Institute for Health Metrics and Evaluation, University of Washington, Seattle, WA, USA.

2. Big Data Institute, University of Oxford, Oxford, UK.

3. Antai College of Economics, Shanghai Jiao Tong University,

Shanghai, China.

4. Montreal Neurological Institute, McGill University, Montreal, QC, Canada.

5. Advanced Diagnostic and Interventional Radiology Research Center,

Tehran University of Medical Sciences, Tehran, Iran.

6. Department of Pediatric Dentistry, Federal University of Minas Gerais, Belo Horizonte, Brazil.

7. Department of Clinical Sciences, University of Sharjah, Sharjah, United Arab Emirates.

8. Department of Internal Medicine, Manipal Academy of Higher

Education, Mangalore, India.

9. Hamadan University of Medical Sciences, Hamadan, Iran.

10. Department of Public Health, Wolkite University, Wolkite, Ethiopia.

11. School of Medicine, Griffith University, Gold Coast, QLD, Australia.

12. Department of Population Health Sciences, King's College London,

London, England.

13. Centre of Excellence for Epidemiological Modeling and Analysis,

Stellenbosch University, Stellenbosch, South Africa.

14. Department of Global Health, Stellenbosch University, Cape Town, South Africa.

15. Center for Policy, Population \& Health Research, National Autonomous

University of Mexico, Mexico City, Mexico.

16. The Australian Centre for Public and Population Health Research (ACPP

HR), University of Technology Sydney, Sydney, NSW, Australia.

17. Lincoln Medical School, Universities of Nottingham \& Lincoln, Lincoln, UK.

18. Department of Epidemiology, Jimma University, Jimma, Ethiopia.

19. Australian Center for Precision Health, University of South Australia,

Adelaide, SA, Australia.

20. Institute of Health Research, University of Health and Allied Sciences, Ho, Ghana.

21. Health Management and Economics Research Center, Iran University of Medical Sciences, Tehran, Iran.

22. Health Economics Department, Iran University of Medical Sciences, Tehran, Iran.

23. Department of Epidemiology, Arak University of Medical Sciences, Arak, Iran.

24. Research Group in Health Economics, University of Cartagena, Cartagena, Colombia.

25. Research Group in Hospital Management and Health Policies, ALZAK

Foundation, Cartagena, Colombia.

26. Carol Davila University of Medicine and Pharmacy, Bucharest, Romania.

27. Statistics and Econometrics Department, Bucharest University of Economic Studies, Bucharest, Romania.

28. Department of Parasitology, Mazandaran University of Medical Sciences, Sari, Iran.

29. Department of Parasitology, Iranshahr University of Medical Sciences, Iranshahr, Iran.

30. Department of Psychology, Foundation University Islamabad,

Rawalpandi, Pakistan.

31. Department of Public Health, Birmingham City University,

Birmingham, UK.

32. Department of Plastic Surgery, University of Texas, Houston, TX, USA.

33. Department of Health System and Health Economics, Bahir Dar University, Bahir Dar, Ethiopia.

34. Department of Forensic Medicine, Lumbini Medical College, Palpa, Nepal. 35. The Judith Lumley Centre, La Trobe University, Melbourne, VIC, Australia. 
36. Department of Community Medicine, Manipal Academy of Higher Education, Manipal, India.

37. Unit of Biochemistry, Sultan Zainal Abidin University, Kuala Terengganu, Malaysia.

38. Department of Hypertension, Medical University of Lodz, Lodz, Poland.

39. Polish Mothers' Memorial Hospital Research Institute, Lodz, Poland.

40. Department of Midwifery, Bahir Dar University, Bahir Dar, Ethiopia.

41. Department of Neurosciences, Costa Rican Department of Social Security, San Jose, Costa Rica.

42. School of Medicine, University of Costa Rica, San Pedro, Costa Rica.

43. Center for Primary Care, Harvard University, Boston, MA, USA.

44. School of Public Health, Imperial College London, London, UK.

45. Department of Community Medicine, Gandhi Medical College Bhopal,

Bhopal, India.

46. Jazan University, Jazan, Saudi Arabia.

47. Department of Medicine, El Bosque University, Bogota, Colombia.

48. Transplant Service, University Hospital Foundation Santa Fe de Bogotá,

Bogota, Colombia.

49. Department of Internal Medicine, University of São Paulo, São

Paulo, Brazil.

50. Department of Internal Medicine, Bahir Dar University, Bahir Dar, Ethiopia.

51. One Health, University of Nantes, Nantes, France.

52. Institute of Public Health, United Arab Emirates University, Hradec Kralova, United Arab Emirates.

53. Department of Social and Clinical Pharmacy, Charles University, Al

Ain, Czech Republic.

54. Department of Anatomy, Government Medical College Pali, Pali, India.

55. Department of Community Medicine and Family Medicine, All India

Institute of Medical Sciences, Jodhpur, India.

56. School of Public Health, All India Institute of Medical Sciences,

Jodhpur, India.

57. Department of Statistical and Computational Genomics, National Institute of Biomedical Genomics, Kalyani, India.

58. Department of Statistics, University of Calcutta, Kolkata, India.

59. Centre for Global Child Health, University of Toronto, Toronto, ON,

Canada.

60. Centre of Excellence in Women and Child Health, Aga Khan University,

Karachi, Pakistan.

61. Social Determinants of Health Research Center, Babol University of

Medical Sciences, Babol, Iran.

62. University of Bologna, Bologna, Italy.

63. Liaison of Turkey, Guillain-Barre Syndrome/Chronic Inflammatory Demyelinating Polyneuropathy Foundation International, Conshohocken, PA, USA. 64. Nutrition Department, St. Paul's Hospital Millennium Medical College,

Addis Ababa, Ethiopia.

65. Department of Veterinary Medicine, Islamic Azad University,

Kermanshah, Iran.

66. Department of Psychiatry, University of São Paulo, São Paulo, Brazil.

67. School of Public Health and Health Systems, University of Waterloo,

Waterloo, ON, Canada.

68. Al Shifa School of Public Health, Al Shifa Trust Eye Hospital,

Rawalpindi, Pakistan.

69. Department of Health Care, Metropolitan Autonomous University, Mexico City, Mexico

70. Research Unit on Applied Molecular Biosciences (UCIBIO), University of

Porto, Porto, Portugal.

71. Colombian National Health Observatory, National Institute of Health,

Bogota, Colombia.

72. Epidemiology and Public Health Evaluation Group, National University of Colombia, Bogota, Colombia.

73. Department of Pharmacology, All India Institute of Medical Sciences, Jodhpur, India.

74. Department of Microbiology \& Infection Control, Medanta Medicity,

Gurugram, India.

75. Department of Medicine, University of Toronto, Toronto, ON, Canada.

76. Department of Public Health, Texila American University,

Georgetown, Guyana.

77. Maternal and Child Health Division, International Centre for Diarrhoeal

Disease Research, Dhaka, Bangladesh.

78. Department of Epidemiology and Biostatistics, University of South

Carolina, Columbia, SC, USA.
79. Department of Pulmonary Medicine, Christian Medical College and Hospital (CMC), Vellore, India.

80. Hanoi National University of Education, Hanoi, Vietnam.

81. Environmental Health Department, Imam Abdulrahman Bin Faisal

University, Dammam, Saudi Arabia.

82. Department of Population and Development, Latin American Faculty of Social Sciences Mexico, Mexico City, Mexico.

83. Department of Public Health, National University of Colombia,

Bogota, Colombia.

84. Department of Medical Laboratory Sciences, Bahir Dar University, Bahir Dar, Ethiopia.

85. Center for Nutrition and Health Research, National Institute of Public Health, Cuernavaca, Mexico.

86. Wellcome Trust Brighton and Sussex Centre for Global Health Research, Brighton and Sussex Medical School, Brighton, UK.

87. School of Public Health, Addis Ababa University, Addis Ababa, Ethiopia.

88. National Centre for AIDS and STD Control, Save the Children, Kathmandu,

Nepal.

89. Department of Global Public Health, Karolinska Institute, Stockholm,

Sweden.

90. Department of Community Medicine, University of Peradeniya,

Peradeniya, Sri Lanka.

91. Department of Health Metrics Sciences, School of Medicine, University of Washington, Seattle, WA.

92. Department of Microbiology, Far Western University, Mahendranagar,

Nepal.

93. Center of Complexity Sciences, National Autonomous University of

Mexico, Mexico City, Mexico.

94. Faculty of Veterinary Medicine and Zootechnics, Autonomous University of Sinaloa, Culiacán Rosales, Mexico.

95. Development of Research and Technology Center, Ministry of Health and Medical Education, Tehran, Iran.

96. School of Medicine, Federal University of Bahia, Salvador, Brazil.

97. Department of Internal Medicine, Escola Bahiana de Medicina e Saúde

Pública (Bahiana School of Medicine and Public Health), Salvador, Brazil.

98. Department of Global Health and Social Medicine, Harvard University,

Boston, MA, USA.

99. Department of Social Services, Tufts Medical Center, Boston, MA, USA

100. Centre for Clinical Epidemiology and Biostatistics, University of

Newcastle, Newcastle, NSW, Australia.

101. Department of Clinical Pathology, Mansoura University, Cairo, Egypt.

102. Pediatric Dentistry and Dental Public Health Department, Alexandria

University, Alexandria, Egypt.

103. School of Behavioral Sciences, Mahatma Gandhi University of Medical

Sciences and Technology, Kottayam, India.

104. Department of Neurology, Cairo University, Cairo, Egypt.

105. Department of Health Policy and Administration, University of the Philippines Manila, Manila, Philippines.

106. Department of Psychology, Federal University of Sergipe, São Cristóvão, Brazil.

107. Research Center for Environmental Determinants of Health, Kermanshah University of Medical Sciences, Kermanshah, Iran.

108. College of Medicine and Public Health, Flinders University, Adelaide, SA, Australia.

109. Associated Laboratory for Green Chemistry (LAQV), University of Porto, Porto, Portugal.

110. Psychiatry Department, Kaiser Permanente, Fontana, CA, USA.

111. School of Health Sciences, A.T. Still University, Mesa, AZ, USA.

112. Institute of Gerontological Health Services and Nursing Research,

Ravensburg-Weingarten University of Applied Sciences, Weingarten,

Germany.

113. Institute of Gerontology, National Academy of Medical Sciences of Ukraine, Kyiv, Ukraine.

114. Department of Medical Parasitology, Abadan Faculty of Medical

Sciences, Abadan, Iran.

115. Department of Dermatology, Kobe University, Kobe, Japan.

116. Department of Cardiovascular Medicine, Cleveland Clinic, Cleveland, $\mathrm{OH}$, USA.

117. Gillings School of Global Public Health, University of North Carolina

Chapel Hill, Chapel Hill, NC, USA.

118. Department of Human Nutrition, Aksum University, Mekelle, Ethiopia. 
119. Department of Nursing and Midwifery, Addis Ababa University, Addis Ababa, Ethiopia.

120. Department of Nursing, Aksum University, Aksum, Ethiopia.

121. Department of Nursing, Mekelle University, Mekelle, Ethiopia.

122. Department of Epidemiology, Mekelle University, Mekelle, Ethiopia.

123. Infectious Disease Research Center, Kermanshah University of Medical

Sciences, Kermanshah, Iran.

124. Pediatric Department, Kermanshah University of Medical Sciences, Kermanshah, Iran.

125. Student Research Committee, Iran University of Medical Sciences, Tehran, Iran.

126. Faculty of Allied Health Sciences, The University of Lahore, Lahore, Pakistan.

127. Health Systems and Policy Research, Indian Institute of Public Health Gandhinagar, Gandhinagar, India.

128. School of Medicine, University of Milan, Milan, Italy.

129. Center for Clinical and Epidemiological Research, University of São

Paulo, Porto Alegre, Brazil.

130. Epidemiology Department, Federal University of Rio Grande do Sul, São Paulo, Brazil.

131. Department of Microbiology, Saint James School of Medicine, The Valley, Anguilla.

132. Department of Epidemiology, Saint James School of Medicine, The Valley, Anguilla.

133. Department of Preventive and Social Medicine, Federal University of Minas Gerais, Belo Horizonte, Brazil.

134. Institute of Tropical Pathology and Public Health (IPTSP), Federal University of Goias, Goiânia, Brazil.

135. Department of Epidemiology and Preventive Medicine, Monash University, Melbourne, VIC, Australia.

136. Department of Epidemiology, Binzhou Medical University, Yantai City, China.

137. Medical Resources, March of Dimes, Arlington, VA, USA.

138. Health Policy, Management and Leadership, West Virginia University School of Public Health, Morgantown, WV, USA.

139. Department of Social and Public Health, Ohio University, Athens, OH, USA.

140. Department of Pharmacology, Tehran University of Medical Sciences,

Tehran, Iran

141. Obesity Research Center, Shahid Beheshti University of Medical Sciences, Tehran, Iran

142. Department of Radiology and Radiological Sciences, Johns Hopkins University, Baltimore, MD, USA

143. University Institute of Public Health, The University of Lahore, Pakistan.

144. Department of Epidemiology, Universitas Airlangga (Airlangga

University), Surabaya, Indonesia.

145. Department of Dental Public Health, Airlangga University, Surabaya, Indonesia.

146. Australian Research Centre for Population Oral Health, University of Adelaide, Adelaide, SA, Australia.

147. Gastrointestinal and Liver Diseases Research Center, Guilan University of Medical Sciences, Rasht, Iran.

148. Caspian Digestive Disease Research Center, Guilan University of Medical Sciences, Rasht, Iran

149. School of Nursing and Midwifery, Tabriz University of Medical Sciences, Tabriz, Iran.

150. Institute of Pharmaceutical Sciences, University of Veterinary and Animal Sciences, Lahore, Pakistan.

151. Department of Pharmacy Administration and Clinical Pharmacy, Xian Jiaotong University, Xian, China.

152. School of Business, London South Bank University, London, UK.

153. Department of Urban Planning and Design, University of Hong Kong, Hong Kong, China.

154. Kasturba Medical College, Manipal Academy of Higher Education,

Manipal, India.

155. Institute of Research and Development, Duy Tan University, Da Nang, Vietnam.

156. Department of Computer Science, University of Human Development, Sulaymaniyah, Iraq.

157. College of Science and Engineering, Hamad Bin Khalifa University, Doha, Qatar.
158. Department of Occupational Safety and Health, China Medical University, Taichung, Taiwan.

159. Department of Public Health and Disease Control, Ministry of Health, Umuahia, Nigeria.

160. Nigerian Field Epidemiology and Laboratory Training Program, African Field Epidemiology Network, Abuja, Nigeria.

161. Department of Health Promotion and Education, University of Ibadan, Ibadan, Nigeria.

162. Department of Community Medicine, University of Ibadan, Ibadan,

Nigeria.

163. Department of Community Medicine, University College Hospital,

Ibadan, Ibadan, Nigeria.

164. Department of Epidemiology, University of Kragujevac, Belgrade, Serbia.

165. University of Belgrade, Kragujevac, Serbia.

166. College of Public Health, Taipei Medical University, Taipei, Taiwan.

167. Department of Internal Medicine, Cleveland Clinic, Cleveland, OH, USA.

168. N. A. Semashko Department of Public Health and Healthcare, I.M.

Sechenov First Moscow State Medical University, Moscow, Russia.

169. Department of Global Health, Economics and Policy, University of

Kragujevac, Kragujevac, Serbia.

170. Department of Community Medicine, Dr. Baba Saheb Ambedkar Medical College \& Hospital, Delhi, India.

171. Department of Community Medicine, Banaras Hindu University, Varanasi, India.

172. Tabriz University of Medical Sciences, Tabriz, Iran.

173. Institute for Prevention of Non-communicable Diseases, Qazvin University of Medical Sciences, Qazvin, Iran.

174. Health Services Management Department, Qazvin University of Medical Sciences, Qazvin, Iran.

175. Department of Forensic Medicine and Toxicology, All India Institute of Medical Sciences, Jodhpur, India.

176. Institute for Epidemiology and Social Medicine, University of Münster, Münster, Münster, Germany.

177. Social Determinants of Health Research Center, Tabriz University of Medical Sciences, Tabriz, Iran.

178. Department of Midwifery, Hawassa University, Hawassa, Ethiopia.

179. International Research Center of Excellence, Institute of Human Virology Nigeria, Abuja, Nigeria.

180. Julius Centre for Health Sciences and Primary Care, Utrecht University, Utrecht, Netherlands.

181. Mashhad University of Medical Sciences, Mashhad, Iran.

182. School of Food and Agricultural Sciences, University of Management

and Technology, Lahore, Pakistan.

183. Department of Epidemiology and Biostatistics, Health Services Academy, Mymensingh, Pakistan.

184. Department of Medical Microbiology \& Immunology, United Arab Emirates University, Islamabad, United Arab Emirates.

185. Department of Population Sciences, Jatiya Kabi Kazi Nazrul Islam University, Al Ain, Bangladesh.

186. Faculty of Health and Medicine, University of Newcastle,

Newcastle, NSW, Australia.

187. Faculty of Health and Wellbeing, Sheffield Hallam University, Sheffield, UK.

188. College of Arts and Sciences, Ohio University, Zanesville, OH, USA.

189. Department of Public Health, Kermanshah University of Medical

Sciences, Kermanshah, Iran.

190. School of Traditional Chinese Medicine, Xiamen University Malaysia,

Sepang, Malaysia.

191. Department of Nursing and Health Promotion, Oslo Metropolitan University, Oslo, Oslo, Norway.

192. School of Health Sciences, Kristiania University College, Oslo, Norway.

193. Global Community Health and Behavioral Sciences, Tulane University, New Orleans, LA, USA.

194. Independent Consultant, Jakarta, Indonesia.

195. Kasturba Medical College, Udupi, India.

196. CIBERSAM, San Juan de Dios Sanitary Park, Sant Boi de Llobregat, Spain.

197. Department of Anthropology, Panjab University, Chandigarh, India.

198. Department of Demography, University of Montreal, Montreal, QC,

Canada.

199. Department of Social and Preventive Medicine, University of Montreal, Montreal, QC, Canada. 
200. Department of Clinical Risk Management, Porto Alegre Clinical Hospital, Porto Alegre, Brazil.

201. Department of Psychiatry, University of Nairobi, Nairobi, Kenya. 202. Division of Psychology and Language Sciences, University College London, London, UK.

203. Department of Medicine, McMaster University, Coventry, ON, Canada. 204. Institute of Occupational and Environmental Medicine, University of Birmingham, Hamilton, UK.

205. Imperial College Business School, Imperial College London, London, UK. 206. Faculty of Public Health, University of Indonesia, Depok, Indonesia. 207. Department of Clinical Sciences and Community Health, University of Milan, Milan, Italy.

208. Public Health Foundation of India, Gurugram, India.

209. Unit of Genetics and Public Health, Institute of Medical Sciences, Las Tablas, Panama.

210. Department of Otorhinolaryngology, Father Muller Medical College, Mangalore, India.

211. School of Nursing, Hong Kong Polytechnic University, Hong Kong, China.

212. Department of Sociology, Shenzhen University, Shenzhen, China.

213. School of Public Health and Preventive Medicine, Monash University, Melbourne, VIC, Australia.

214. Department of Systems, Populations, and Leadership, University of Michigan, Ann Arbor, MI, USA.

215. Department of Pharmaceutical Science, University of Eastern Piedmont, Novara, Italy.

216. Chemistry Department, Salahaddin University-Erbil, Erbil, Iraq.

217. Center for Integration of Data and Health Knowledge, Oswald Cruz Foundation (FIOCRUZ), Salvador, Brazil.

218. Centre for Global Mental Health (CGMH), London School of Hygiene \& Tropical Medicine, London, England.

219. National Center for the Prevention and Control of HIV and AIDS, National Institute of Health, Mexico City, Mexico.

220. Department of Maternal and Child Nursing and Public Health, Federal University of Minas Gerais, Belo Horizonte, Brazil.

221. Department of Epidemiology and Biostatistics, Tehran University of Medical Sciences, Tehran, Iran.

222. Department of Nursing, Majmaah University, Majmaah, Saudi Arabia.

223. Research Group, Foundation for the Promotion of Life (Fundovida IPS), Cartagena, Colombia.

224. Campus Caucaia, Federal Institute of Education, Science and Technology of Ceará, Caucaia, Brazil.

225. Department of Midwifery, Hamadan University of Medical Sciences, Hamadan, Iran.

226. ICF International, DHS Program, Rockville, MD, USA.

227. Department of Dentistry, Autonomous University of Hidalgo State, Pachuca, Mexico.

228. College of Medicine, Alfaisal University, Riyadh, Saudi Arabia.

229. Research \& Innovation Center, Ministry of Health, Riyadh, Saudi Arabia.

230. Peru Country Office, United Nations Population Fund (UNFPA), Lima,

Peru.

231. Forensic Medicine Division, Imam Abdulrahman Bin Faisal University, Dammam, Saudi Arabia.

232. Clinical Microbiology and Parasitology Unit, Dr. Zora Profozic Polyclinic, Zagreb, Croatia.

233. University Centre Varazdin, University North, Varazdin, Croatia.

234. Departament of Statistics and Econometrics, Bucharest University of Economic Studies, Bucharest, Romania.

235. Heidelberg Institute of Global Health (HIGH), Heidelberg University, Heidelberg, Germany.

236. Institute of Addiction Research (ISFF), Frankfurt University of Applied Sciences, Frankfurt, Germany.

237. Internal Medicine Department, King Saud University, Riyadh, Saudi Arabia.

238. Department of Biostatistics, Hamadan University of Medical Sciences, Hamadan, Iran.

239. Department of Epidemiology and Biostatistics, Shahrekord University of Medical Sciences, Shahrekord, Iran.

240. Kashmar Center of Higher Health Education, Mashhad University of Medical Sciences, Mashhad, Iran.
241. Health Systems and Policy Research Unit, Ahmadu Bello University, Zaria, Nigeria.

242. University of New South Wales, NSW, Sydney, Australia.

243. School of Public Health, Iran University of Medical Sciences,

Kermanshah, Iran.

244. Computer, Electrical, and Mathematical Sciences and Engineering

Division, King Abdullah University of Science and Technology, Thuwal, Saudi Arabia.

245. Department of Food Science, University of Campinas (Unicamp),

Campinas, Brazil.

246. Department of Pediatric Medicine, The Children's Hospital \& The Institute of Child Health, Multan, Pakistan.

247. Department of Pediatrics \& Pediatric Pulmonology, Institute of Mother \& Child Care, Multan, Pakistan.

248. Prasanna School of Data Science, Manipal Academy of Higher

Education, Manipal Udupi, India.

249. Research and Analytics Department, Initiative for Financing Health and Human Development, Chennai, India.

250. Department of Research and Analytics, Bioinsilico Technologies,

Chennai, India.

251. Laboratory of Public Health Indicators Analysis and Health Digitalization, Moscow Institute of Physics and Technology, Dolgoprudny, Russia.

252. Experimental Surgery and Oncology Laboratory, Kursk State Medical University, Kursk, Russia.

253. Department of Clinical Medicine, Federal University of Minas Gerais, Belo Horizonte, Brazil.

254. Clinical Hospital, Federal University of Minas Gerais, Belo Horizonte, Brazil.

255. Department of Biotechnology, University of Central Punjab, Lahore, Pakistan.

256. Department of Forensic Medicine and Toxicology, Manipal Academy of Higher Education, Manipal, India.

257. Department of Pediatrics, Arak University of Medical Sciences, Arak, Iran. 258. Medical Laboratory Sciences, Adigrat University, Adigrat, Ethiopia.

259. Department of General Surgery, Carol Davila University of Medicine and Pharmacy, Bucharest, Romania.

260. Department of General Surgery, Emergency Hospital of Bucharest, Bucharest, Romania.

261. Department of Community Medicine, Kathmandu University, Palpa, Nepal.

262. Department of Public Health, University of Yaoundé I, Yaoundé,

Cameroon.

263. Institute for Global Health Innovations, Duy Tan University, Hanoi, Vietnam.

264. Centre for Heart Rhythm Disorders, University of Adelaide, Adelaide, WA, Australia.

265. Unit of Microbiology and Public Health, Institute of Medical Sciences, Las Tablas, Panama.

266. Department of Public Health, Ministry of Health, Herrera, Panama. 267. Administrative and Economic Sciences Department, University of Bucharest, Bucharest, Romania.

268. Translational Health Research Institute, Western Sydney University, Sydney, NSW, Australia.

269. Department of Psychiatry and Behavioral Neurosciences, McMaster University, Hamilton, ON, Canada.

270. Department of Psychiatry, University of Lagos, Lagos, Nigeria.

271. Department of Respiratory Medicine, Jagadguru Sri Shivarathreeswara

Academy of Health Education and Research, Mysore, India.

272. Corporate, Somnogen Canada Inc., Toronto, ON, Canada.

273. Department of Economic Geography, National Autonomous University of Mexico, Mexico City, Mexico.

274. Department of Neurology and Public Health, Icahn School of Medicine at Mount Sinai, New York, NY, USA.

275. Department of Genetics, Yale University, New Haven, CT, USA.

276. School of Global Public Health, New York University, New York, NY, USA.

277. Laboratory of Genetics and Molecular Cardiology, University of São

Paulo, São Paulo, Brazil.

278. Department of Genetics, Harvard University, Boston, MA, USA.

279. Center for Integration of Data and Health Knowledge, Oswaldo Cruz Foundation, Salvador, Brazil. 
280. HIV and Mental Health Department, Integrated Development Foundation Nepal, Kathmandu, Nepal.

281. University Medical Center Groningen, University of Groningen, Groningen, Netherlands.

282. School of Economics and Business, University of Groningen, Groningen, Netherlands.

283. Department of Pharmacology, Imam Abdulrahman Bin Faisal University, Dammam, Saudi Arabia.

284. Clinical Research Center, Fundación Valle del Lili, Cali, Colombia.

285. Center for Studies in Social Protection and Health Economics, ICESI University, Cali, Colombia.

286. National Institute of Infectious Diseases, Bucharest, Romania.

287. Department of Infectious Diseases, Carol Davila University of Medicine and Pharmacy, Bucharest, Romania.

288. Health Sciences Department, Muhammadiyah University of Surakarta, Sukoharjo, Indonesia.

289. College of Medicine, University of Central Florida, Orlando, FL, USA

290. Thalassemia and Hemoglobinopathy Research Center, Ahvaz

Jundishapur University of Medical Sciences, Ahvaz, Iran.

291. Metabolomics and Genomics Research Center, Tehran University of

Medical Sciences, Tehran, Iran.

292. Department of Community Medicine, Maharishi Markandeshwar

Institute of Medical Sciences \& Research, Solan, India.

293. Department of Computer Science, Khazar University, Baku, Azerbaijan.

294. Department of Pharmacology, Shahid Beheshti University of Medical

Sciences, Tehran, Iran.

295. Department of Public Health, North South University, Dhaka,

Bangladesh.

296. Department of Biostatistics and Epidemiology, University of

Massachusetts Amherst, Amherst, MA, USA.

297. Research Department, Policy Research Institute, Kathmandu, Nepal.

298. Health and Public Policy Department, Global Center for Research and

Development, Kathmandu, Nepal.

299. Department of Oral Pathology, Srinivas Institute of Dental Sciences,

Mangalore, India.

300. Department of Primary Care and Public Health, Imperial College

London, London, UK.

301. Academic Public Health England, Public Health England, London, UK.

302. WHO Collaborating Centre for Public Health Education and Training,

Imperial College London, London, UK.

303. University College London Hospitals, London, UK.

304. Department of Computer Science, Boston University, Boston, MA, USA.

305. School of Nursing and Midwifery, Royal College of Surgeons in Ireland Bahrain, Muharraq Governorate, Bahrain.

306. Research Center for Immunodeficiencies, Tehran University of Medical Sciences, Tehran, Iran.

307. Network of Immunity in Infection, Malignancy and Autoimmunity (NIIM A), Universal Scientific Education and Research Network (USERN), Tehran,

Iran.

308. Epidemiology Research Unit Institute of Public Health (EPIUnit-ISPUP), University of Porto, Porto, Portugal.

309. Department of Clinical Research, Federal University of Uberlândia, Uberlândia, Brazil.

310. Center for Research in Congenital Anomalies and Rare Diseases, ICESI University, Cali, Colombia.

311. Malaria Atlas Project, University of Oxford, Oxford, UK.

312. Department of Health Statistics, National Institute for Medical Research

Dar es Salaam, Tanzania.

313. Department of Internal Medicine, University of Botswana, Gaborone, Botswana.

314. Department of Psychiatry, All India Institute of Medical Sciences, New Delhi, India.

315. Department of Phytochemistry, Soran University, Soran, Iraq.

316. Department of Nutrition, Cihan University-Erbil, Erbil, Iraq.

317. Public Health and Community Medicine Department, Cairo University, Giza, Egypt.

318. Department of Entomology, Ain Shams University, Cairo, Egypt.

319. Department of Health and Society, University of Applied and

Environmental Sciences, Bogota, Colombia.

320. National School of Public Health, Carlos III Health Institute, Madrid, Spain.
321. Department of Geriatrics and Long Term Care, Hamad Medical Corporation, Doha, Qatar.

322. Faculty of Health \& Social Sciences, Bournemouth University, Bournemouth, UK.

323. School of Health Sciences, Federal University of Santa Catarina, Araranguá, Brazil.

324. Department of Population and Health, University of Cape Coast, Cape Coast, Ghana.

325. College of Public Health, Medical and Veterinary Sciences, James Cook University, Townsville, QLD, Australia.

326. Center for Biomedical Information Technology, Shenzhen Institutes of Advanced Technology, Shenzhen, China.

327. Independent Consultant, Karachi, Pakistan.

328. Centre for Medical Informatics, University of Edinburgh, Edinburgh, UK.

329. Division of General Internal Medicine, Harvard University, Boston, MA,

USA.

330. Institute for Population Health, King's College London, London, UK.

331. College of Medicine, Yonsei University, Seoul, South Korea.

332. Department of Physical Education, Federal University of Santa Catarina,

Florianópolis, Brazil.

333. School of Medicine, University of Alabama at Birmingham, Birmingham, $\mathrm{AL}$, USA.

334. US Department of Veterans Affairs (VA), Birmingham, AL, USA.

335. Department No.16, Moscow Research and Practical Centre on

Addictions, Moscow, Russia.

336. Therapeutic Department, Balashiha Central Hospital, Balashikha, Russia.

337. Nursing Care Research Center, Semnan University of Medical Sciences, Semnan, Iran.

338. Department of Community Medicine, Ahmadu Bello University, Zaria, Nigeria.

339. Department of Biomedical Sciences, Arba Minch University, Arba

Minch, Ethiopia.

340. Health Education and Behavioral Science, University of Gondar,

Gondar, Ethiopia.

341. School of Public Health, Mekelle University, Mekelle, Ethiopia.

342. Southgate Institute for Health and Society, Flinders University,

Adelaide, SA, Australia.

343. Department of Pathology and Legal Medicine, University of São Paulo, Ribeirão Preto, Brazil.

344. Modestum LTD, London, UK.

345. Department of Health Economics, Hanoi Medical University,

Hanoi, Vietnam.

346. College of Medicine and Health Sciences, Bahir Dar University, Bahir

Dar, Ethiopia.

347. Department of Community Medicine, Alex Ekwueme Federal University Teaching Hospital Abakaliki, Abakaliki, Nigeria.

348. Department of Medical and Surgical Sciences, University of Bologna,

Bologna, Italy.

349. Occupational Health Unit, Sant'Orsola Malpighi Hospital, Bologna, Italy.

350. Ho Chi Minh City University of Technology (HUTECH), Ho Chi Minh

City, Vietnam

351. Center of Excellence in Behavioral Medicine, Nguyen Tat Thanh

University, Ho Chi Minh City, Vietnam.

352. Foundation University Medical College, Foundation University Islamabad, Islamabad, Pakistan

353. School of Population Health \& Environmental Sciences, King's College London, London, UK

354. Department of Midwifery, Adigrat University, Adigrat, Ethiopia.

355. Competence Center of Mortality-Follow-Up of the German National Cohort, Federal Institute for Population Research, Wiesbaden, Germany. 356. Department of Community Medicine, Rajarata University of Sri Lanka, Anuradhapura, Sri Lanka.

357. School of International Development and Global Studies, University of Ottawa, Ottawa, ON, Canada.

358. The George Institute for Global Health, University of Oxford, Oxford, UK. 359. Centre for Suicide Research and Prevention, University of Hong Kong, Hong Kong, China.

360. Department of Social Work and Social Administration, University of Hong Kong, Hong Kong, China.

361. Department of Neuropsychopharmacology, National Center of Neurology and Psychiatry, Kodaira, Japan. 
362. Department of Public Health, Juntendo University, Tokyo, Japan. 363. Department of Epidemiology and Biostatistics, Wuhan University, Wuhan, China.

364. Global Health Institute, Wuhan University, Wuhan, China. 365. Cancer Institute, Hacettepe University, Ankara, Turkey. 366. Department of Health Care Management and Economics, Urmia University of Medical Science, Urmia, Iran.

367. Laboratory of Genetics and Genomics, Moscow Research and Practical Centre on Addictions, Moscow, Russia.

368. Addictology Department, Russian Medical Academy of Continuous Professional Education, Moscow, Russia.

369. School of Medicine, Wuhan University, Wuhan, China.

370. School of Public Health, Wuhan University of Science and Technology, Wuhan, China.

371. Hubei Province Key Laboratory of Occupational Hazard Identification and Control, Wuhan University of Science and Technology, Wuhan, China. 372. Department of Health Education \& Promotion, Kermanshah University of Medical Sciences, Kermanshah, Iran.

\section{Authors' contributions}

Providing data or critical feedback on data sources: JA, NA, HA, LGA, EAG, BA, MaA, AEA, VA, OOA, MA-B, BOA, KA, MBA, RKA, VaA, AAH, NAG, RA, TA, DA, MuA, JalA, OA, MA, MAJ, AA, BPAQ, SA, DBM, AAB, MacB, SiB, MAB, SaB, MB, $\mathrm{DBB}, \mathrm{NeB}, \mathrm{DBR}, \mathrm{IB}, \mathrm{TB}, \mathrm{FB}, \mathrm{YB}, \mathrm{ASB}, \mathrm{PB}, \mathrm{NB}, \mathrm{KB}, \mathrm{ZAB}, \mathrm{AIB}, \mathrm{SMB}, \mathrm{ZWB}, \mathrm{SB}, \mathrm{AB}$, $A R B, Z B, A C G, R C, F C, J M C M, C C O, J C, S C, S K C, V K C, M A K C, D J C, D T C, A J C$, MC, NC, AC, ACP, SMAD, FD, CDC, NDW, BNDG, FDH, FMD, EDG, KebD, KD, SDD, GD, DD, SD, AD, LDL, RE, AWE, LE, AE, MESZ, MET, SEJ, EJF, AF, NF, NKF, $E F, P F, I F, F F, N A F, M F, S F R, T F, M M G, G G, H A G, K G, A G, S A G, E G, T G, M G, H G$, MDCG, RAG, YG, RG, EH, MRH, TH, AH-M, AsH, AH, NH, SH, HH, SIH, KH, NJH, $\mathrm{CH}, \mathrm{HCH}, \mathrm{RH}, \mathrm{MH}, \mathrm{MOH}, \mathrm{BFH}, \mathrm{Cl}, \mathrm{SEl}, \mathrm{OI}, \mathrm{MI}, \mathrm{II}, \mathrm{U}, \mathrm{DJ}, \mathrm{VJ}, \mathrm{MJ}, \mathrm{AJN}, \mathrm{RPJ}, \mathrm{KJ}, \mathrm{NJ}$, FJ, LRK, R, TK, BeKM, AnK, SEK, GK, AKTT, GAK, AKK, MK, NaK, EK, GuK, MNK, KhK, NK, YJK, SezK, AdK, SK, SLKL, AK, KK, BKD, RK, VK, DNK, ManK, OK, DK, CLV, DL, HLN, IL, SL, PL, KLG, IDL, ShL, BL, XL, HIMA, DM, CMR, DCM, MoAM, MDM, CMA, FRMM, SZM, BKM, CEMS, ZAM, WM, RGM, TM, AM, BM, Ymoh, NM, SMM, AMH, ReM, SM, AHM, MAM, MM, YM, RM, PM, AMK, GM, LM, RavN, MDN, BN, MN, VCN, JN, HN, IN, SN, GNT, RN, JJN, VNS, BO, FO, ATO, NO, SO, MPA, JRP, SRPP, APM, UP, EP, SP, JP, KP, MP, FHP, SIP, LLP, DRAP, AR, FR, MHUR, AMR, KR, JR, DCLR, SJR, PR, SR, DLR, RR, VR, NR, AzR, AIR, LR, ER, GR, RS, SMS, MS, AMS, RSS, BS, LS, IS, A-AS, ALS, FS, MAS, KiS, AzS, KS, JIS, KHS, DASS, JS, VS, AAS, AmS, AS, SS, KMS, MBS, CTN, FW, RT, RLT, GTV, MRTP, BT, GT, CU, BU, JVH, YV, FSV, BV, YasW, YPW, YW, PW, SW, RW, NW, MY, SY, PY, NY, CY, DY, HY, MZ, MSZ, ZJZ, YZ, AZ. Development of methods or computational machinery: MBA, AAH, DA, SA, ASB, SB, DJC, MC, AC, FD, LD-L, LE, NJH, $\mathrm{MH}, \mathrm{LRK}, \mathrm{SEK}, \mathrm{NK}, \mathrm{AdK}, \mathrm{KLG}, \mathrm{AHM}, \mathrm{JN}, \mathrm{RN}, \mathrm{DCLR}, \mathrm{AMS}, J \mathrm{VH}, \mathrm{YV}$, AZ. Providing critical feedback on methods or results: NA, HA, LGA, EAG, BA, AEA, VA, OOA, BOA, KA, MBA, RKA, VaA, AAH, NAG, TA, DA, MuA, JalA, OA, AA, BPAQ, SA, $\mathrm{DBM}, \mathrm{MacB}, \mathrm{SiB}, \mathrm{MAB}, \mathrm{SB}, \mathrm{DBB}, \mathrm{DBR}, \mathrm{IB}, \mathrm{FB}, \mathrm{YB}, \mathrm{ASB}, \mathrm{PB}, \mathrm{NB}, \mathrm{ZAB}, \mathrm{AIB}, \mathrm{SMB}$, ZWB, SoB, AB, ARB, ZB, ACG, RC, JMCM, JC, SC, SC, VKC, MAKC, DJC, DTC, MC, AdCP, SMAD, CDC, BNDG, FMD, KebD, KD, DD, SD, LD-L, RE, AWE, AE, MESZ, EJF, AF, NF, FF, NAF, MF, SFR, TF, MMG, HAG, AG, SAG, EG, TG, MG, HG, YG, $\mathrm{RG}, \mathrm{EH}, \mathrm{MRH}, \mathrm{TH}, \mathrm{AsH}, \mathrm{NH}, \mathrm{SH}, \mathrm{SIH}, \mathrm{KH}, \mathrm{NJH}, \mathrm{CH}, \mathrm{HCH}, \mathrm{RH}, \mathrm{MH}, \mathrm{MoH}, \mathrm{BFH}, \mathrm{Cl}$, SEI, OI, MI, II, UI, VJ, MJ, AJN, RPJ, FJ, LRK, RK, TK, BeKM, AnK, SEK, GK, AyK, GAK, MK, NaK, EK, GuK, MNK, KhK, YJK, AdK, SezK, SLKL, AK, KK, BKD, RK, VK, DNK, ManK, OK, DK, CLV, DL, HLN, SL, KLG, ShL, BL, XL, CMR, DCM, MoAM, MDM, CMA, FRMM, SZM, BKM, CEMS, ZAM, WM, RGM, TM, AM, BM, Ymoh, NM, AMH, SM, AHM, MAM, RM, AMK, GM, LM, RavN, MDN, BN, MN, JN, HN, IN, SN, GNT, RN, JJN, BO, FO, ATO, NO, MPA, JRP, SRPP, APM, UP, EP, JP, KP, MP, FHP, SIP, DRAP, AR, FR, JR, DCLR, SJR, PR, SR, VR, NR, AzR, AIR, LR, GR, RS, SMS, MS, AMS, RSS, BS, LS, IS, A-AS, FS, MAS, KiS, AzS, KS, JIS, DASS, JS, VS, AAS, AS, MBS, CTN, FiT, FT, RT, GTV, MRTP, BT, GT, CU, BU, JVH, YV, FSV, YasW, YPW, YW, PW, RW, NW, SY, PY, NY, CY, DY, HY, MZ, MSZ, YZ, AZ. Drafting the manuscript or revising it critically for important intellectual content: NA, HA, LGA, EAG, BA, AEA, VA, OOA, BOA, KA, MBA, RKA, VaA, AAH, NAG, RA, TA, DA, MuA, JalA, OA, MA, AA, BPAQ, SA, DBM, AAB, MacB, SiB, MAB, SB, DBB, NeB, $\mathrm{DBR}, \mathrm{IB}, \mathrm{TB}, \mathrm{FB}, \mathrm{YB}, \mathrm{ASB}, \mathrm{PB}, \mathrm{NB}, \mathrm{ZAB}, \mathrm{AIB}, \mathrm{SMB}, \mathrm{ZWB}, \mathrm{SOB}, \mathrm{AB}, \mathrm{ARB}, \mathrm{ZB}, \mathrm{ACG}$, RC, FC, JMCM, CCO, JC, SC, SKC, VKC, MAKC, DJC, DTC, MC, NC, ACP, SMAD, CDC, NDW, BNDG, FDH, FMD, EDG, KebD, KD, SDD, GD, DD, SD, LD-L, RE, AWE, AE, MESZ, MET, SEJ, EJF, AF, NF, NKF, PF, FF, NAF, MF, SFR, TF, MMG, $\mathrm{GG}, \mathrm{HAG}, \mathrm{AG}, \mathrm{SAG}, \mathrm{EG}, \mathrm{TG}, \mathrm{MG}, \mathrm{HG}, \mathrm{MDCG}, \mathrm{RAG}, \mathrm{YG}, \mathrm{RG}, \mathrm{EH}, \mathrm{MRH}, \mathrm{TH}, \mathrm{AsH}$, $\mathrm{AH}, \mathrm{NH}, \mathrm{SH}, \mathrm{SIH}, \mathrm{KH}, \mathrm{NJH}, \mathrm{CH}, \mathrm{HCH}, \mathrm{RH}, \mathrm{MH}, \mathrm{MoH}, \mathrm{BFH}, \mathrm{Cl}, \mathrm{SEl}, \mathrm{OI}, \mathrm{MI}, \mathrm{II}, \mathrm{UI}, \mathrm{VJ}$ MJ, AJN, RPJ, NJ, FJ, LRK, RK, TK, BeKM, AnK, SEK, GK, AK, GAK, MK, NaK, EK,
GuK, MNK, KhK, YJK, SezK, AdK, SLKL, AK, KK, BKD, RK, VK, DNK, ManK, OK, DK, CLV, DL, HLN, IL, SL, PL, KLG, ShL, BL, XL, DM, CMR, DCM, MoAM, MDM, CMA, FRMM, SZM, BKM, CEMS, ZAM, WM, RGM, TM, AM, BM, YMoh, NM, AMH, SM, AHM, MAM, MM, RM, PM, AMK, GM, LM, RavN, MDN, BN, MN, VCN, JN, HN, IN, SN, GNT, RN, JJN, VNS, BO, FO, ATO, NO, MPA, JRP, SRPP, APM, UP, ShP, $E P, J P, K P, M P, F H P, S I P, A R, F R, M H \cup R$, JR, DCLR, SJR, PR, SR, VR, NR, AzR, AIR, LR, GR, RS, SMS, MS, AMS, RSS, BS, LS, IS, A-AS, FS, MAS, KiS, AzS, KS, JIS, DASS, JS, VS, AAS, AS, MBS, CTN, RT, RLT, GTV, MRTP, BT, GT, CU, BU, JVH, YV, FSV, YasW, YPW, YW, PW, RW, NW, MY, SY, PY, NY, CY, DY, HY, MZ, MSZ, YZ, AZ Management of the overall research enterprise (for example, through membership in the Scientific Council): YV, SB, AHM, MA, LD-L, BN, MPA, MESZ, NR, RS, SIH, YM, TG, AJC, SP, AS. All authors read and approved the final manuscript.

\section{Funding}

This work was primarily supported by grant OPP1132415 from the Bill \& Melinda Gates Foundation. The funder of the study had no role in study design, data collection, data analysis, data interpretation, writing of the report, or decision to publish. The corresponding authors had full access to all the data in the study and had final responsibility for the decision to submit for publication.

\section{Availability of data and materials}

Our study follows the Guidelines for Accurate and Transparent Health Estimates Reporting (GATHER). Estimates can be further explored at national, state, and municipality level by age group, sex, and year through our online visualization tools (https://vizhub.healthdata.org/lbd/hiv-mort-la). The source code used to generate estimates, as well as the outputs of the study (including full sets of estimates at the state and municipality levels), are publicly available online via the Global Health Data Exchange (http://ghdx. healthdata.org/record/ihme-data/latin-america-hiv-mortality-estimates-2000-2 017). All maps presented in this study were generated by the authors and no permissions are required to publish them.

\section{Ethics approval and consent to participate} Not applicable.

\section{Consent for publication}

Not applicable.

\section{Competing interests}

Dr. Singh reports personal fees from Crealta/Horizon, Medisys, Fidia, UBM LLC, Trio health, Medscape, WebMD, Clinical Care options, Clearview healthcare partners, Putnam associates, Focus forward, Navigant consulting, Spherix, Practice Point communications, the National Institutes of Health and the American College of Rheumatology, personal fees from Simply Speaking, other from Amarin, Viking, Moderana and Vaxart pharmaceuticals, nonfinancial support from FDA Arthritis Advisory Committee, non-financial support from Steering committee of OMERACT, an international organization that develops measures for clinical trials and receives arm's length funding from 12 pharmaceutical companies, non-financial support from Veterans Affairs Rheumatology Field Advisory Committee, non-financial support from Editor and the Director of the UAB Cochrane Musculoskeletal Group Satellite Center on Network Meta-analysis, outside the submitted work. Dr. Krishan reports grants from UGC Centre of Advanced Study, CAS II, awarded to the Department of Anthropology, Panjab University, Chandigarh, India, outside the submitted work. Prof. Postma reports grants and personal fees from various pharmaceutical industries, all outside the submitted work. Prof Postma holds stocks in Ingress Health and Pharmacoeconomics Advice Groningen (PAG Ltd) and is advisor to Asc Academics, all pharmacoeconomic consultancy companies. Dr. Ancuceanu reports he received consultancy and speakers' fees from various pharmaceutical companies. Walter Mendoza is a Program Analyst in Population and Development at the United Nations Population Fund-UNFPA Country Office in Peru, an institution which does not necessarily endorse this study. Dr. Pandi-Perumal reports a non-financial relationship with Somnogen Canada Inc. and occasional royalities from publishing houses, outside the submitted work. 
Received: 31 August 2020 Accepted: 27 November 2020 Published online: 08 January 2021

\section{References}

1. Roth GA, Abate D, Abate KH, Abay SM, Abbafati C, Abbasi N, et al. Global, regional, and national age-sex-specific mortality for 282 causes of death in 195 countries and territories, 1980-2017: a systematic analysis for the Global Burden of Disease Study 2017. Lancet. 2018;392:1736-88.

2. Frank TD, Carter A, Jahagirdar D, Biehl MH, Douwes-Schultz D, Larson SL, et al. Global, regional, and national incidence, prevalence, and mortality of HIV, 1980-2017, and forecasts to 2030, for 195 countries and territories: a systematic analysis for the Global Burden of Diseases, Injuries, and Risk Factors Study 2017. Lancet HIV. 2019;6:e831-59.

3. Fast-Track - Ending the AIDS epidemic by 2030. https://www.unaids.org/en/ resources/documents/2014/JC2686 WAD2014report. Accessed 24 Apr 2020.

4. Ravasi G, Grinsztejn B, Baruch R, Guanira JV, Luque R, Cáceres CF, et al. Towards a fair consideration of PrEP as part of combination HIV prevention in Latin America. J Int AIDS Soc. 2016;19 7Suppl 6. doi:https://doi.org/10. 7448/IAS.19.7.21113.

5. Joint United Nations Programme on HIV/AIDS. AIDSinfo. https://aidsinfo. unaids.org/. Accessed 24 Apr 2020.

6. Luz PM, Veloso VG, Grinsztejn B. The HIV epidemic in Latin America: accomplishments and challenges on treatment and prevention. Curr Opin HIV AIDS. 2019;14:366-73.

7. García Jl, Sabidó M, Nikiforov M, Smith A, Hernández G, Ortiz R, et al. The UALE project: a cross-sectional approach for trends in HIV/STI prevalence among key populations attending STI clinics in Guatemala. BMJ Open. 2018; 8:e022632.

8. Kerr LR, Kendall C, Guimarães M, Mota R, Veras MA, Dourado I, et al. HIV prevalence among men who have sex with men in Brazil: results of the 2 nd national survey using respondent-driven sampling. Medicine. 2018;97:S9-15.

9. Colchero M, Romero M, Conde-Glez C, Sosa-Rubí S. Is the HIV epidemic stable among MSM in Mexico? HIV Prevalence and Risk Behavior Results from a Nationally Representative Survey among Men Who Have Sex with Men. Plos one. 2013;8:e72616.

10. Guimarães ML, Marques BCL, Bertoni N, Teixeira SLM, Morgado MG, Bastos $\mathrm{Fl}$, et al. Assessing the HIV-1 epidemic in Brazilian drug users: a molecular epidemiology approach. Plos One. 2015;10:e0141372.

11. Hebert JR, Hurley TG, Chiriboga DE, Barone J. A comparison of selected nutrient intakes derived from three diet assessment methods used in a lowfat maintenance trial. Public Health Nutr. 1998;1:207-14.

12. Local Burden of Disease Child Growth Failure Collaborators. Mapping child growth failure across low- and middle-income countries. Nature. 2020;577: 231-4.

13. Sgarbi RVE, Carbone A da SS, Paião DSG, Lemos EF, Simionatto S, Puga MAM, et al. A Cross-Sectional Survey of HIV Testing and Prevalence in Twelve Brazilian Correctional Facilities Plos One 2015;10:e0139487.

14. Local Burden of Disease Educational Attainment Collaborators. Mapping disparities in education across low- and middle-income countries. Nature. 2020;577:235-8.

15. Ross J, Henry N, Dwyer-Lindgren L, Lobo A, Marinho de Souza MDF, Biehl $M$, et al. Progress toward eliminating TB and HIV deaths in Brazil, 2001-2015: A spatial assessment. BMC Med 2018;1:1-10.

16. Alves ATJ, Nobre FF. The acquired immunodeficiency syndrome in the state of Rio de Janeiro, Brazil: a spatio-temporal analysis of cases reported in the period 2001-2010. Geospat Health. 2014;8:437-43.

17. de Holanda ER, Galvão MTG, Pedrosa NL, Paiva S de S, de Almeida RLF. Spatial analysis of infection by the human immunodeficiency virus among pregnant women. Rev Lat Am Enfermagem 2015;23:441-449.

18. Tuboi SH, Schechter M, McGowan CC, Cesar C, Krolewiecki A, Cahn P, et al. Mortality during the first year of potent antiretroviral therapy in HIV-1infected patients in 7 sites throughout Latin America and the Caribbean. J Acquir Immune Defic Syndr. 2009;51:615-23.

19. Pletcher. Model fittiing and hypothesis testing for age specific data. J Evol Biol. 2001;12:430-9.

20. Alexander M, Zagheni E, Barbieri M. A flexible Bayesian model for estimating subnational mortality. Demography. 2017;54:2025-41.

21. Ocaña-Riola R, Mayoral-Cortes JM. Spatio-temporal trends of mortality in small areas of southern Spain. BMC Public Health. 2010;10:26.

22. Dwyer-Lindgren L, Squires E, Teeple S, Ikilezi G, Roberts D, Colombara D, et al. Small area estimation of under-5 mortality in Bangladesh, Cameroon,
Chad, Mozambique, Uganda, and Zambia using spatially misaligned data. Popul Health Metrics. 2018;16:13.

23. Murray CJL, Ortblad KF, Guinovart C, Lim SS, Wolock TM, Roberts DA, et al. Global, regional, and national incidence and mortality for HIV, tuberculosis, and malaria during 1990-2013: a systematic analysis for the Global Burden of Disease Study 2013. Lancet. 2014;384:1005-70.

24. Carvalho CN, Dourado I, Bierrenbach AL. Underreporting of the tuberculosis and AIDS comorbidity: an application of the linkage method. Rev Saude Publica. 2011;45:548-55

25. Birnbaum J, Murray C, Lozano R. Exposing misclassified HIV/AIDS deaths in South Africa. Bull World Health Organ. 2011;89:278-85.

26. Lima EEC, Queiroz BL, Zeman K. Completeness of birth registration in Brazil: an overview of methods and data sources. Genus. 2018;74 https://doi.org/ 10.1186/s41118-018-0035-9.

27. Mikkelsen L, Phillips DE, AbouZahr C, Setel PW, de Savigny D, Lozano R, et al. A global assessment of civil registration and vital statistics systems: monitoring data quality and progress. Lancet. 2015;386:1395-406.

28. Bhat P. Completeness of India's sample registration system: an assessment using the general growth balance method. Popul Stud. 2002;56:119-34.

29. Murray C, Rajaratnam J, Marcus J, Laakso T, Lopez A. What can we conclude from death registration? Improved Methods for Evaluating Completeness. Plos Med. 2010;7:e1000262.

30. Adair T, Lopez AD. Estimating the completeness of death registration: an empirical method. PLoS One. 2018;13 https://doi.org/10.1371/journal.pone. 0197047.

31. Schmertmann CP, Gonzaga MR. Bayesian estimation of age-specific mortality and life expectancy for small areas with defective vital records. Demography. 2018;55:1363-88.

32. Stevens GA, Alkema L, Black RE, Boerma JT, Collins GS, Ezzati M, et al. Guidelines for accurate and transparent health estimates reporting: the GATHER statement. Lancet. 2016;388:e19-23.

33. World Health Organization. ICD-10: International statistical classification of diseases and related health problems. 1992.

34. Naghavi M, Makela S, Foreman K, O'Brien J, Pourmalek F, Lozano R. Algorithms for enhancing public health utility of national causes-of-death data. Popul Health Metrics. 2010;8:9.

35. Global Administrative Unit Layers (GAUL). http://www.fao.org/geonetwork/ srv/en/metadata.show?id=12691. Accessed 27 May 2020

36. Humanitarian Data Exchange. https://data.humdata.org/. Accessed 27 May 2020.

37. ArcMap. https://desktop.arcgis.com/en/arcmap/. Accessed 14 Jun 2020.

38. WorldPop. https://www.worldpop.org/project/categories?id=3. Accessed 17 Mar 2020.

39. Earth Observation Group - Defense Meteorological Satellite Progam, Boulder | ngdc.noaa.gov. https://www.ngdc.noaa.gov/eog/dmsp/downloadV4 composites.html. Accessed 17 Mar 2020.

40. Global Human Settlement - Data overview - European Commission. https://ghsl.jrc.ec.europa.eu/data.php. Accessed 17 Mar 2020.

41. Weiss D, Nelson A, Gibson H, Temperley W, Peedell S, Lieber A, et al. A global map of travel time to cities to assess inequalities in accessibility in 2015. Nature. 2018;553:333-36.

42. Evans CAW, Friedland JS, Gotuzzo E. HIV in South America. Medicine (Abingdon). 2005;33:44-5.

43. Burstein R, Henry N, Collison M, Marczak L, Sligar A, Watson S, et al. Mapping 123 million neonatal, infant and child deaths between 2000 and 2017. Nature. 2019;574:353-8.

44. Målqvist M, Eriksson L, Nga N, Fagerland L, Hoa D, Ewald U, et al. Unreported births and deaths, a severe obstacle for improved neonatal survival in low-income countries; a population based study. BMC Int Health Hum Rights. 2008;8:4.

45. Dwyer-Lindgren L, Bertozzi-Villa A, Stubbs RW, Morozoff C, Kutz MJ, Huynh $C$, et al. US county-level trends in mortality rates for major causes of death, 1980-2014. JAMA. 2016;316:2385-401.

46. Kristensen K, Nielsen A, Berg CW, Skaug H, Bell BM. TMB: automatic differentiation and Laplace approximation. J Stat Softw. 2016;70:1-21.

47. R Core Team. R: a language and environment for statistical computing. Vienna: Foundation for Statistical Computing; 2015.

48. Alonso Gonzalez M, Martin L, Munoz S, Jacobson JO. Patterns, trends and sex differences in HIV/AIDS reported mortality in Latin American countries: 1996-2007. BMC Public Health. 2011;11:605. 
49. Bozon M, Gayet C, Barrientos J. A life course approach to patterns and trends in modern Latin American sexual behavior. J Acquir Immune Defic Syndr. 2009;51(Suppl 1):S4-12.

50. Miller WM, Buckingham L, Sánchez-Domínguez MS, Morales-Miranda S, PazBailey G. Systematic review of HIV prevalence studies among key populations in Latin America and the Caribbean. Salud Publica Mex. 2013; 55(Suppl 1):S65-78.

51. Brito AM, Kendall C, Kerr L, Mota RMS, Guimarães MDC, Dourado I, et al. Factors associated with low levels of HIV testing among men who have sex with men (MSM) in Brazil. PLoS One. 2015;10:e0130445.

52. Kerrigan D, Vazzano A, Bertoni N, Malta M, Bastos Fl. Stigma, discrimination and HIV outcomes among people living with HIV in Rio de Janeiro, Brazil: the intersection of multiple social inequalities. Glob Public Health. 2017;12: 185-99

53. Dolan K, Kite B, Black E, Aceijas C, Stimson G. HIV in prison I low-income and middle-income countries. Lancet Infect Dis. 2007;7:32-41.

54. Prellwitz IM, Alves BM, Ikeda MLR, Kuhleis D, Picon PD, Jarczewski CA, et al. HIV behind bars: human immunodeficiency virus cluster analysis and drug resistance in a reference correctional unit from Southern Brazil. Plos One. 2013;8 https://doi.org/10.1371/journal.pone.0069033.

55. Crabtree-Ramírez B, Belaunzarán-Zamudio PF, Cortes CP, Morales M, Sued O, Sierra-Madero J, et al. The HIV epidemic in Latin America: a time to reflect on the history of success and the challenges ahead. J Int AIDS Soc. 2020;23 https://doi.org/10.1002/jia2.25468.

56. Venezuela | UNAIDS. https://www.unaids.org/en/regionscountries/countries/ venezuela. Accessed 27 Jul 2020.

57. Benzaken AS, Pereira GFM, Costa L, Tanuri A, Santos AF, Soares MA. Antiretroviral treatment, government policy and economy of HIV/AIDS in Brazil: is it time for HIV cure in the country? AIDS Res Ther. 2019;16:19.

58. Mexico | UNAIDS. https://www.unaids.org/en/regionscountries/countries/ mexico. Accessed 18 Nov 2020.

59. Prachniak-rincón C. Villar de Onís J. HIV and the right to health in Colombia Health Hum Rights. 2016;18:157-69.

60. Barrington C, Knudston K, Bailey OAP, Aguilar JM, Loya-Montiel MI, MoralesMiranda S. HIV diagnosis, linkage to care, and retention among men who have sex with men and transgender women in Guatemala City. J Health Care Poor Underserved. 2016;27:1745-60.

61. HIV prevention in the SPOTLIGHT. An analysis from the perspective of the health sector in Latin America and the Caribbean. https://iris.paho.org/ bitstream/handle/10665.2/34381/9789275119792-eng.pdf?sequence $=$ 6\&isAllowed=y. Accessed 18 Nov 2020.

62. Cabezas MC, Fornasini M, Dardenne N, Borja T, Albert A. A crosssectional study to assess knowledge about HIV/AIDS transmission and prevention measures in company workers in Ecuador. BMC Public Health. 2013;13:139.

63. Ecuador | UNAIDS. https://www.unaids.org/en/regionscountries/countries/ ecuador. Accessed 18 Nov 2020.

64. Carriquiry G, Fink V, Koethe JR, Giganti MJ, Jayathilake K, Blevins M, et al. Mortality and loss to follow-up among HIV-infected persons on long-term antiretroviral therapy in Latin America and the Caribbean. J Int AIDS Soc. 2015;18:20016

65. Sierra-Madero JG, Belaunzaran-Zamudio PF, Crabtree-Ramírez B, MagisRodriguez C. Mexico's fragmented health system as a barrier to HIV care Lancet HIV. 2019;6:e74-5.

66. García PJ, Bayer A, Cárcamo CP. The changing face of HIV in Latin America and the Caribbean. Curr HIV/AIDS Rep. 2014;11:146-57.

67. Đào LU, Terán E, Bejarano S, Hernandez I, Reina Ortiz M, Chee V, et al. Risk and resiliency: the syndemic nature of HIV/AIDS in the indigenous highland communities of Ecuador. Public Health. 2019;176:36-42.

68. Caro-Vega Y, Belaunzarán-Zamudio PF, Crabtree-Ramírez B, Shepherd BE, Mejia F, Giganti MJ, et al. Trends in proportion of older HIV-infected people in care in Latin America and the Caribbean: a growing challenge. Epidemiol Infect. 2018;146:1308

69. Lima M da S, Firmo AAM, Martins-Melo FR. Trends in AIDS-related mortality among people aged 60 years and older in Brazil: a nationwide populationbased study. AIDS Care. 2016;28:1533-40.

70. Mpondo BCT. HIV infection in the elderly: arising challenges. J Aging Res. 2016:2016 https://doi.org/10.1155/2016/2404857.

71. Nash D, Katyal M, Brinkhof MWG, Keiser O, May M, Hughes R, et al. Longterm immunologic response to antiretroviral therapy in low-income countries: collaborative analysis of prospective studies. AIDS. 2008;22:2291 302.

72. Hoagland B, Moreira RI, De Boni RB, Kallas EG, Madruga JV, Vasconcelos R, et al. High pre-exposure prophylaxis uptake and early adherence among men who have sex with men and transgender women at risk for HIV infection: the PrEP Brasil demonstration project. J Int AIDS Soc. 2017:20: 21472.

73. Gomez GB, Borquez A, Caceres CF, Segura ER, Grant RM, Garnett GP, et al. The potential impact of pre-exposure prophylaxis for HIV prevention among men who have sex with men and transwomen in Lima, Peru: a mathematical modelling study. Plos Med. 2012:9:e1001323.

74. UNAIDS. Global AIDS update 2018: Miles to go 2018. https://www.unaids. org/sites/default/files/media_asset/miles-to-go_en.pdf. Accessed 4 Jun 2019.

75. Anderson S-J, Cherutich P, Kilonzo N, Cremin I, Fecht D, Kimanga D, et al. Maximising the effect of combination HIV prevention through prioritisation of the people and places in greatest need: a modelling study. Lancet. 2014; 384:249-56.

76. Gerberry DJ, Wagner BG, Garcia-Lerma JG, Heneine W, Blower S. Using geospatial modelling to optimize the rollout of antiretroviral-based preexposure HIV interventions in Sub-Saharan Africa. Nature Commun. 2014;5: ncomms6454

77. Stevens FR, Gaughan AE, Linard C, Tatem AJ. Disaggregating census data for population mapping using random forests with remotely-sensed and ancillary data. Plos One. 2015;10:e0107042. https://doi.org/10.1371/journal. pone.0107042.

\section{Publisher's Note}

Springer Nature remains neutral with regard to jurisdictional claims in published maps and institutional affiliations.
Ready to submit your research? Choose BMC and benefit from:

- fast, convenient online submission

- thorough peer review by experienced researchers in your field

- rapid publication on acceptance

- support for research data, including large and complex data types

- gold Open Access which fosters wider collaboration and increased citations

- maximum visibility for your research: over $100 \mathrm{M}$ website views per year

At BMC, research is always in progress.

Learn more biomedcentral.com/submissions 